\title{
WILEY-VCH
}

DOI: $10.1002 /$ ((please add manuscript number))

Article type: Full Paper

\section{Iron Oxide Nanoparticle-Based Magnetic Ink Development for Fully Printed Tunable Radio-Frequency Devices}

Mohammad Vaseem*, Farhan Abdul Ghaffar, Muhammad Fahad Farooqui, and Atif Shamim

Dr. M. Vaseem, Dr. F. A. Ghaffar, Dr. M. F. Farooqui, Prof. A. Shamim

King Abdullah University of Science and Technology (KAUST), IMPACT Lab, Computer, Electrical and Mathematical Sciences and Engineering (CEMSE) Division, Thuwal 23955-

6900, Kingdom of Saudi Arabia

E-mail: mohammad.vaseem@kaust.edu.sa

Keywords: Iron Oxide Nanoparticle-based Ink, Inkjet Printing, Magnetic Properties, RF Characterization, Tunable Devices

The field of printed electronics is still in its infancy and most of the reported work is based on commercially available nanoparticle-based metallic inks. Although fully printed devices that employ dielectric/semiconductor inks have recently been reported, there is a dearth of functional inks that can demonstrate controllable devices. The lack of availability of functional inks is a barrier to the widespread use of fully printed devices. For radio-frequency (RF) electronics, magnetic materials have many uses in reconfigurable components but rely on expensive and rigid ferrite materials. A suitable magnetic ink can facilitate the realization of fully printed, magnetically controlled, tunable devices. This report presents the development of an iron oxide nanoparticle-based magnetic ink. First, a tunable inductor is fully printed using iron oxide nanoparticle-based magnetic ink. Furthermore, iron oxide nanoparticles were functionalized with oleic acid to make them compatible with a UV-curable SU8 solution. Functionalized iron oxide nanoparticles were successfully embedded in the SU8 matrix to make magnetic substrate. The as-fabricated substrate was characterized for its magnetostatic and microwave properties. A frequency tunable printed patch antenna is demonstrated using the magnetic and in-house silver-organo-complex (SOC) inks. This is a step towards low-cost, fully printed, controllable electronic components. 


\section{WILEY-VCH}

\section{Introduction}

Since printing technology presents a low-cost, high-throughput, and completely digital fabrication process, it is becoming popular with electronics manufacturing. ${ }^{[1]}$ The roll-to-roll manufacturing capability of printing makes it a viable option for mass production to meet the medium-to-large volume production requirements. Several reports have described the development of conductors, dielectric and semiconductor inks for transistors, ${ }^{[2-4]}$ photovoltaic ${ }^{[5-6]}$ memory devices, ${ }^{[7-9]}$ sensors, ${ }^{[10-12]}$ biological devices, ${ }^{[13-14]}$ and radio-frequency (RF) electronics ${ }^{[15-17]}$. However, there are few reports of fully inkjet-printed devices. ${ }^{[6-9,18-19]}$ For fully printed components and devices, different materials inks must be developed since this field is still immature. Fully printed microwave components were recently demonstrated by combining 3D inkjet printing of dielectrics with $2 \mathrm{D}$ printing of metallic inks. ${ }^{[20]}$ The next generation of fully printed components and systems should have the ability to control their performance, such that they can be tuned or reconfigured when necessary; this requires the development of functional inks that are magnetic, ferroelectric, or piezoelectric.

In RF electronics, tunable or reconfigurable components are becoming important due to the proliferation of new wireless devices, different wireless standards in different parts of the world, and high congestion in the existing bands of wireless communication. Furthermore, magnetic materials have been used effectively for tunable and reconfigurable components such as inductors, antennas, and phase shifters. ${ }^{[21-25]}$. Recently, many such designs have been shown in multilayer ferrite LTCC (Low Temperature Co-fired Ceramic) technology. ${ }^{[26-30]}$ But, LTCC technology is quite expensive and it will be really neat if the same things can be done through printing technologies. However, there is a paucity of functional inks with magnetic properties and few reports on magnetic ink-printing. Hoseon Lee et al. ${ }^{[31]}$ demonstrated inkjet printing of commercially available, cobalt-based, ferromagnetic nanoparticles $(\sim 200 \mathrm{~nm})$ for the miniaturization of flexible printed inductors. These metallic cobalt nanoparticles usually 


\section{WILEY-VCH}

require surface passivation to avoid the oxidation problem. Han Song et al. ${ }^{[32]}$ utilized an interesting approach to align the cobalt nanoparticle ink with an external magnetic field during printing to enable prototyping and development of novel, magnetic, composite materials and components. Murali Bissannagari et al. ${ }^{[33]}$ inkjet-printed NiZn-ferrite films using NiZn-ferrite nanoparticle-based ink, completing its magnetic characterization. All the above inks are metallic in nature, but a magnetic ink with dielectric (insulator) properties is required for tunable RF applications. Though there is a commercial magnetic ink solution available from Sigma Aldrich ${ }^{[34]}$, it has a low concentration $(<1 \mathrm{wt} \%)$ of iron oxide nanoparticles and is not suitable for these RF applications. Thus, no tunable or reconfigurable, fully printed RF component based on magnetic ink has been reported to date.

This paper first presents an iron oxide nanoparticle-based magnetic ink, which has been completely characterized for its material properties, and then demonstrates its utility through fully printed, magnetically controllable RF devices. A simple solution method to synthesizing well-dispersed, uniform, magnetic, iron oxide NPs was adopted. These iron oxide NPs were used for ink-formulation and then to demonstrate the fully inkjet printed tunable inductor. These iron oxide nanoparticles were also used with the aim of making free-standing magnetic substrates. Inspired by the 3D-printing technology where polymeric resin materials are used that can immediately solidify on exposure to a low-cost UV lamp, SU8 polymer was selected to develop thick substrates. ${ }^{[35]}$ This SU8 solution can be cross-linked through polymerization by UV-exposure to make thick solid films. Through functionalization of iron oxide nanoparticles with oleic acid (to make it compatible with a UV-curable SU8 solution), the functionalized iron oxide nanoparticles were successfully embedded in the SU8 matrix, creating a free-standing, magnetic substrate. The magnetic ink was characterized for its magnetic and high frequency properties. Finally, a patch antenna was printed on the magnetic substrate with an in-house silver ink; the printed antenna can be tuned for its frequency by 


\section{WILEY-VCH}

applying magnetic fields across it. This first demonstration of a fully printed controllable RF device is an important milestone for the next generation of low-cost tunable and reconfigurable components that can be completely realized through additive manufacturing.

\section{Results and Discussion}

\subsection{Synthesis and Functionalization of Iron Oxide NPs}

Iron oxide NPs were prepared at $90{ }^{\circ} \mathrm{C}$ with iron (II) chloride, iron (III) chloride, $\mathrm{NaOH}$, and acetic acid using the hot-injection solution method for 30 minutes, without the use of any complex reagents. The presence of acetic acid and addition of sodium hydroxide at heating temperature played an important role in the formation of small iron oxide NPs. If sodium hydroxide was added to the boiling solution with the presence of acetic acid, higher temperatures generally caused faster reaction rates, generating large amounts of nuclei in a short time and leading to the formation of small nanoparticles. ${ }^{[2]}$ Iron oxide NPs usually possess typical magnetic behavior at room-temperature, which has been widely explored for many applications, either in the form of discrete nanoparticles or thin-film-based devices. ${ }^{[36-}$

${ }^{39]}$ However, to the best of the authors' knowledge, there is no report on iron oxide NP-based ink formulation for inkjet printing. Several significant issues related to magnetic ink formulation must be addressed. For example, magnetic materials should be nanoparticle sized and be well disperse during formulation, ink viscosity and surface tension must be suited for inkjet printing, and ink must contain the appropriate concentration and a carrier vehicle (solvent). In the field of printed electronics, similar to other emerging electronic technologies, new materials and processing methods are required for their continually improving development and performance.

The as-prepared iron oxide NPs show good dispersion with de-ionized (DI) water and were successfully utilized as a solvent for inkjet printing. To be compatible with SU8 polymeric 


\section{WILEY-VCH}

resin, iron oxide requires functionalization on the surfaces of nanoparticles. SU8 2002 manufactured by MicroChem ${ }^{[35]}$ is usually composed of an epoxy that is dissolved into an organic solvent (e.g. cyclopentanone). Oleic acid is well known and has been successfully used as a molecule for functionalization of iron oxide nanoparticles in many published papers, especially in the case of biological applications. ${ }^{[40]}$ The selection of oleic acid is due to its compatibility with cyclopentanone of SU8 polymeric resin in addition to many common organic solvents. Furthermore, the choice of SU8 is also due to its low wt $\%$ of resin with low viscosity solvent composition and its photo-curing capability. A number of other photocurable polymeric resins are available but due to their high content of resin (>99\%) and high viscosity, it is very challenging to embed the nanoparticles in those resins. Thus, for compatibility with SU8 2002, iron oxide nanoparticles were functionalized with oleic acid, as shown in Figure 1a. The physical adsorption of oleic acid molecules on the surfaces of iron oxide NPs leads to compatibility with the SU8 matrix, as the long chain of oleic acid interacts with the organic solvent. Subsequently, these SU8-mixed nanoparticles were pre-heated at $80{ }^{0} \mathrm{C}$ for 15 minutes, followed by UV curing with a wavelength of $365 \mathrm{~nm}$ for 30 minutes to solidify the mixture. Figure $1(b-d)$ and (e-g) shows the scanning electron microscopic (SEM) images and energy dispersive spectroscopic (EDS) spectrum taken from the as-prepared, functionalized, SU8-mixed iron oxide NPs. The SEM image (b \& c) shows that the NPs are almost spherical shape; they are uniformly grown at a high density with an average diameter of 15 to $20 \mathrm{~nm}$. It should be noted that due to charging effect and magnetization of iron oxide nanoparticles, high resolution images were difficult to capture. The EDS spectrum (e) demonstrates that the as-prepared NPs are made of $\mathrm{Fe}$ and $\mathrm{O}$ only, and the atomic ratio of $\mathrm{Fe}$ and $\mathrm{O}$ is approximately $3: 4$. In contrast to pure iron oxide NPs, the oleic acid functionalized sample (f) shows the carbon content in addition to $\mathrm{Fe}$ and $\mathrm{O}$, which confirms the functionalization on the surfaces of iron oxide NPs. The SU8 mixed iron oxide NPs (g) show even higher carbon content, primarily due to SU8 molecules. The SU8-mixed, iron oxide morphology (d) 


\section{WILEY-VCH}

confirms that nanoparticles are well embedded in the SU8 matrix. The particle's size and shape is further confirmed by its corresponding transmission electron microscopic (TEM) images. Figure S1 shows TEM image, high resolution (HR) TEM image, and selected area diffraction (SAED) pattern of (a) pure, (b) functionalized, and (c) SU8-mixed iron oxide NPs. From the TEM images, it is confirmed that nanoparticles are in various shapes such as spherical, cubic and elongated. Such shapes are common in iron oxide nanoparticles during the nucleation and growth formation which has also been confirmed by other researcher. ${ }^{[40]}$ The functionalization of iron oxide is also visualized by an HR-TEM image (b2) that shows $\sim 2$ to $3 \mathrm{~nm}$ of carbon shell on the core of the nanoparticles. Furthermore, the HR-TEM image (c2) of the SU8-mixed sample shows a thick boundary of the SU8 carbon-coated matrix, in which iron oxide nanoparticles are suspended. The SAED patterns (a3-c3) for all the samples, confirmed the poly-crystallinity phase of as-grown iron oxide NPs, correspond to the cubic spinel structure. Due to thick boundary of SU8 matrix, the intensity of SAED pattern is faded which is further conformed that iron oxide nanoparticles embedded in the SU8 matrix. It should be noted that TEM analyses are only for morphological characterization purpose. However, functionalization and embedding could be efficiently confirmed by FTIR analysis.

\subsection{Chemical Nature and Crystalline Phase of Iron Oxide NPs}

The quality and chemical composition of (a) as-synthesized, (b) oleic acid functionalized, and (c) SU8-mixed iron oxide NPs were further examined by Fourier transform infrared (FTIR) spectroscopy in transmission mode and are shown in Figure 2 (i). In the as-synthesized sample, weak adsorption bands appeared at 3412 and $1587 \mathrm{~cm}^{-1}$ as well as a strong adsorption band at $581 \mathrm{~cm}^{-1}$. The weak adsorptions bands could be attributed to the stretching vibration and bending vibration of the absorbed water and surface hydroxyls, respectively. [41] Moreover, the presence of the strong band is due to Fe-O stretching vibration. Surface absorbed moisture is common during sample preparation for FT-IR analysis. Therefore, the 


\section{WILEY-VCH}

FTIR spectrum confirmed that the synthesized product was pure iron oxide NP (a). The bands at 1395 and $1458 \mathrm{~cm}^{-1}$ can be ascribed to the symmetric and asymmetric stretches of $\mathrm{COO}^{-}$, indicating that the oleic acid molecule is attached to the iron oxide nanoparticles in a bidentate mode, with two oxygen atoms symmetrically coordinated to iron (b). ${ }^{[42]}$ The characteristic vibrational bands at 2852 and $2920 \mathrm{~cm}^{-1}$ are attributed to the symmetric and antisymmetric $\mathrm{CH}_{2}$ stretching from the structure of oleic acid. ${ }^{[42]}$ In addition, the band at $1639 \mathrm{~cm}^{-1}$ is due to $\mathrm{C}=\mathrm{C}$ from oleic acid. The SU8-mixed iron oxide sample shows several characteristic bands at $830,1245,1608$, and $1738 \mathrm{~cm}^{-1}$, which respectively correspond to an epoxide, aromatic ring, and carbonyl group from the SU8 molecules. ${ }^{[43]}$

Figure 2 (ii) shows the X-ray diffraction (XRD) patterns that were implemented to examine the crystal structure of iron oxide nanoparticles (a) before and (b) after functionalization, and (c) SU8-mixed samples. All characteristic peaks are matched with the cubic spinal-structured magnetite (JCPDS card no. 65-3107). ${ }^{[44]}$ The optical properties of the as-synthesized and functionalized samples were also investigated by UV-Vis absorption (supporting information in Figure S2). The UV-Vis absorption spectrum shows that, as the wavelength decreases, absorbance increases monotonically, which is congruent with previously reported iron oxide nanoparticles. ${ }^{[45]}$ The UV-Vis spectrum indicates that the wide absorption range from 300 to $900 \mathrm{~nm}$ occurred with a broad peak center at $400 \mathrm{~nm}$, corresponding to iron oxide absorption. The wide absorption may be caused by cluster formations of iron oxide NPs in an ethanol solution, which scattered almost UV radiation and provided long-tail type features in the UV-Vis absorption spectrum.

\subsection{Magnetic Properties of Iron Oxide NPs}

Figure 3 demonstrates the magnetization versus magnetic field plots ( $\mathrm{M}-\mathrm{H}$ loops) measured at $300 \mathrm{~K}$, in addition to the full range of hysteresis between $\pm 10 \mathrm{k}$ Oe for the assynthesized and oleic acid functionalized iron oxide NPs. The samples show no hysteresis at 


\section{WILEY-VCH}

room-temperature (RT), signifying the superparamagnetic nature of the resultant NPs (a). A well-developed hysteresis loop was observed at $5 \mathrm{~K}$, signifying the ferromagnetic nature of the resultant NPs (b). It is believed that, due to the air-gap condition and loosely bound nanoparticles at RT measurement, the samples are superparamagnetic nature. In contrast to RT measurement, the freezing state (at $5 \mathrm{~K}$ ) condition satisfied the gap and loosely bound state, demonstrating the ferromagnetic nature, as shown with the pictorial presentation in Figure $3 \mathrm{a}$ and $\mathrm{b}$. Furthermore, the saturation magnetization $\left(\mathrm{M}_{\mathrm{s}}\right)$, remanent magnetization $\left(\mathrm{M}_{\mathrm{R}}\right)$, and coercivity $\left(\mathrm{H}_{\mathrm{C}}\right)$ were calculated as $51-53 \mathrm{emu} / \mathrm{g}, 20.31 \mathrm{emu} / \mathrm{g}$, and $400 \mathrm{Oe}$ for assynthesized and oleic acid functionalized iron oxide NPs, respectively (Figure 3b). Compared to the bulk iron oxide, ${ }^{[46]}$ the decrease in $\mathrm{M}_{\mathrm{S}}$ was attributed to the decreased particle size and an increase in surface area.

The energy of a magnetic particle in an external field is proportional to its size or volume via the number of magnetic molecules in a single magnetic domain. When this energy becomes comparable to the thermal energy, thermal fluctuations will significantly reduce the total magnetic moment at a given field. ${ }^{[47]}$ Such a phenomenon is more prominent with small nanocrystals. The temperature-dependent magnetization was also characterized (supporting information in Figure S3), which is typical to magnetic nanoparticles. Zero-field cooled (ZFC) and field-cooled (FC) curves were measured in a magnetic field of 100 Oe in the temperature range of 5 to $300 \mathrm{~K}$. The graph shows the two FC and ZFC curves, which have a divergence point close to room-temperature. Moreover, FC magnetization remains nearly constant as the temperature decreases, whereas ZFC magnetization decreases as the temperature decreases. There is no distinctive blocking temperature $\left(T_{B}\right)$, which must be investigated further by varying the magnetic field.

\subsection{Inkjet Printing of Magnetic Ink and Fabrication of Fully Printed Tunable Inductor}




\section{WILEY-VCH}

As-synthesized iron oxide NPs were used for ink formulation with $10 \mathrm{wt} \%$ loading in water solvent. To confirm the particle size aggregate in the ink, the ink was diluted 10-times with deionized (DI) water and characterized using Zetasizer (as shown in Figure S4). The graph clearly shows the size distribution with number of particles. The $\sim 51.7 \%$ particle aggregates correspond to $121.5 \mathrm{~nm}, 48 \%$ for $242 \mathrm{~nm}$ and $0.2 \%$ for $2582 \mathrm{~nm}$. The ink was inkjet printed under constant printing conditions and vertically dropped from the nozzle, which formed dots (supporting information Figure S5) and lines (supporting information Figure S6 \& Figure S7) on the glass substrate. The 3D image of the dots shows the uniform dot pattern with a high density of NPs covering the entire dot area (Figure S5 (a)). The 3D surface profiler measured the dot width as $\sim 55 \mu \mathrm{m}$ and the thickness was $\sim 160 \mathrm{~nm}$ (Figure S5 (b)). To further study the effect of overprinting on the substrate-surface-ink interaction and line uniformity, the line patterns with a drop spacing of $40 \mu \mathrm{m}$ were printed on glass substrates while varying the number of over-layers $\left(n_{\mathrm{ol}}\right)$. The as-printed iron oxide lines were analyzed by the 3D surface profiler with varying $n_{\mathrm{ol}}$ (supporting information in Figures S6 \& S7). The printed lines show a width of $70 \pm 10 \mu \mathrm{m}$ with number of over-layers. Figure S6 (a) corresponds to the single printing, which reveals that the density of NPs is not uniform along the width (i.e. there is a higher density at the edge of line than in the middle area; a-2). The first-layer of printing is directly related to the substrate-ink interaction and its compatible properties, such as the surface tension of ink and surface energy of the substrate, direct the quality of printed lines. It is believed that with successive overprinting, one can cover the edge area with more NPs and eventually print a more uniform pattern line. To confirm this, we have overprinted layer over the first-printed layer. As shown in Figure S6 (b) and (c) and Figure S6, line uniformity and density is substantially improved by increasing the number of over-layers from 1 to 5 . In addition, all the printed lines with the ink did not show any coffee-ring effects or line bulging. The thickness of the as-printed lines with $n_{\mathrm{ol}}$ is summarized in Figure S8. The thicknesses of 


\section{WILEY-VCH}

printed lines can be controlled by the number of overprinted layers and by varying the drop spacing.

In order to evaluate the functional properties of the magnetic ink, a tunable inductor has been fabricated on top of an inkjet printed magnetic film, as shown in Figure 4. First iron oxide nanoparticles ink has been inkjet printed on a plastic substrate with 5-overprinted layers, followed by drying at $80{ }^{\circ} \mathrm{C}$ for $30 \mathrm{~min}$ (Fig. 4(a)). The printed film resulted in a saturation magnetization of approximately 12.4 memu under an applied field of $1 \mathrm{kOe}$, while the coercivity is found to be $46 \mathrm{Oe}$, as shown in supporting information Figure S9 (a). After printing the magnetic film, a total of eight layers of silver-organo-complex (SOC) based silver ink has been printed and cured using infrared (IR) heating for five minutes (Fig. 4(b)). Finally, the fully printed tunable inductor is attached on an FR4 board (support substrate) for testing purpose, as has been shown in (Fig. 4(c)). For RF characterization of the inductor, two port Sparameter measurements have been performed using Agilent E8361C PNA series network analyzer. The inductor is fed by a $50 \Omega$ microstrip transmission line. The measured inductance of the printed inductor is shown in Figure 5. At $100 \mathrm{MHz}$, it has an inductance of $19.6 \mathrm{nH}$ and self-resonant frequency (SRF) of $870 \mathrm{MHz}$ (a). To measure the tunability of the printed inductor, an external magnetic field of up to $12 \mathrm{kOe}$ has been applied using MicroMag ${ }^{\mathrm{TM}}$ 3900 vibrating sample magnetometer (VSM). The inductance versus the frequency under the influence of external magnetic field for printed inductor is shown in Figure 5 (b). A tuning of about $24 \%$ is observed when $12 \mathrm{kOe}$ magnetic field is applied. A smaller tuning of about $18 \%$ is observed when lower magnetic field of $2 \mathrm{kOe}$ is applied. It should be noted that the fully printed inductor realized with our formulated ink shows much higher tuning than the case when commercial iron oxide nanoparticles ink has been used (only $0.8 \%$ tuning with $5 \mathrm{kOe}$ magnetic field, supporting information Figure S10). ${ }^{[34]}$ The results summarized in Table 1 


\section{WILEY-VCH}

clearly indicate superior performance and suitability to tunable RF components as compared to the commercially available ink.

\subsection{Fabrication of Free-standing Magnetic Substrate and its Characterization}

Functionalized iron oxide nanoparticles were successfully embedded in the SU8 matrix to develop free-standing magnetic substrate. The functionalized iron oxide nanoparticles were mixed using stone mortar and pestle with the SU8 2000 (Microchem) epoxy resist at a 50:50 weight $\%$ ratio to formulate ink paste. When the ink paste was ready, it was printed using a manual screen-printing technique (i.e. squeegee). The steps for the fabrication process are displayed in Figure 6. An FR-4 board with a sacrificial paper on the backside was used as a support material in this work, though any other material can be used instead of FR-4. The sacrificial paper was used because the ink was initially in a paste form and a support substrate is required until it solidifies after UV exposure. A slot was created in the support material using LPKF4 Protomat S103 (a) to facilitate the printing of magnetic ink for a precise substrate thickness of $1.5 \mathrm{~mm}$. The empty slot was filled with the ink paste in three cycles. For each cycle, the filled materials were heated to $80{ }^{0} \mathrm{C}$ for 15 minutes followed by UV curing $(\lambda=365 \mathrm{~nm})$ for 15 minutes (b). Once the ink was solidified with three cycles of the heating and the curing process, it was separated from the support material by cutting it from the edges (c). The sacrificial paper on the back of the magnetic substrate was removed by immersing it in warm water for 10 minutes. A $10 \mu \mathrm{m}$ smoothening layer of ' $3 \mathrm{D}$ vero black plus' material ${ }^{[20]}$ was then inkjet printed and photo cured on the top (d) and bottom (e) of the magnetic substrate. A total of eight layers of SOC ink each for ground plane and patch antenna were printed and cured using infrared (IR) heating for five minutes (f). The final prototype of patch antenna is shown in the inset of Figure 6 (f). The preparation of freestanding substrate was performed through manual printing. However, it is envisioned that 


\section{WILEY-VCH}

through integrating advanced printing technology, such as a 3D printer equipped with a UV curing system, in-demand magnetic objects can easily be created.

\subsection{Magnetostatic and Microwave Characterization}

Once the magnetic substrate is prepared, it is important to characterize its magnetostatic and microwave properties. A vibrating sample magnetometer (VSM) was used for the $\mathrm{B}(\mathrm{H})$ curve measurements of the magnetic substrate, where $\mathrm{B}$ is the magnetic flux density and $\mathrm{H}$ is the magnetic field strength. The substrate, without any metallic layers on top, was placed in the VSM; the measured $\mathrm{B}(\mathrm{H})$ curve results are displayed in Figure 7a. The substrate demonstrates a saturation magnetization $\left(4 \pi \mathrm{M}_{\mathrm{S}}\right)$, coercive field $\left(\mathrm{H}_{\mathrm{C}}\right)$, and remanent magnetization $\left(\mathrm{B}_{\mathrm{M}}\right)$ of 1560 Gauss $(\mathrm{G}), 46$ Oe, and 350 Gauss, respectively. For microwave tunable designs, stronger the saturation magnetization, larger is the tunability of the component. Here, the value of $1560 \mathrm{G}$ is acceptable and can provide decent tuning. In the future, this value can be increased by modifying the composition of the ink. After obtaining saturation magnetization, the next important parameter is the magnetization frequency of the substrate. The magnetization frequency is an important characteristic of ferrites because the ferrites do not show any low field losses after this frequency. ${ }^{[48]}$ From the saturation magnetization, the magnetization frequency of the material is calculated using the following formula: $f_{m}=\gamma 4 \pi \mathrm{M}_{\mathrm{S}}=4.37 \mathrm{GHz}$. It is recommended that the center frequency of a microwave device be higher than the magnetization frequency of the substrate to avoid any low field losses. ${ }^{[49]}$

Once the magnetostatic properties of the material are known, it can be studied for its high frequency and microwave properties. To extract the microwave properties of the printed substrate, a coplanar waveguide (CPW) based ring resonator was designed and fabricated on top of the substrate. ${ }^{[50]}$ The resonator was characterized for its $\mathrm{S}$ parameters from $1 \mathrm{GHz}$ to $10 \mathrm{GHz}$, and the fundamental resonance of the design was measured at $2.4 \mathrm{GHz}$. These results 


\section{WILEY-VCH}

have been used to extract the dielectric constant of the material. Since this is a magnetic material, the result obtained from the equation provides a product of the initial permeability and permittivity. This product is displayed in Figure $7 \mathrm{~b}$. The value of this product varies with respect to the frequency, which is expected due to the varying initial permeability of the ferrite material. ${ }^{[51]}$ Using this equation, the permittivity and permeability of the medium at different frequencies were calculated and are listed in Table 2. Since the material is lossy below $\mathrm{f}_{\mathrm{m}}$, frequencies above $\mathrm{fm}$ are considered in the table since these are the frequencies that can be used for the antenna design. In the initial design of any microwave device, this product can directly be used in the equation of the resonant frequency. In addition to the dielectric constant, the loss tangent $(\tan \delta)$ of the material was calculated from the measured results. The conductor losses were calculated using the transmission line calculator of the Keysight in the Advanced Design System (ADS). The measured conductivity of the metal is 5 $\times 10^{6} \mathrm{~S} / \mathrm{m}$, which was used to evaluate the conductor loss for different frequencies. Once the conductor loss was known, the dielectric loss of the material was evaluated. The loss tangent of the substrate at frequencies below $\mathrm{f}_{\mathrm{m}}$ is relatively high. For example, at $2.4 \mathrm{GHz}$ and $4 \mathrm{GHz}$, the loss tangent values are 0.13 and 0.015 respectively, due to the low field losses of the magnetic material in the absence of the magnetic bias. ${ }^{[49]}$ However, the loss tangent values at frequencies above $f_{m}$ are in the acceptable range.

\subsection{Printed Linear Patch Antenna}

The magnetostatic and microwave characteristics of the printed magnetic ink were then used for the design of a patch antenna. The patch antenna design was used as proof of concept to show the viability of this ink in the implementation of tunable and reconfigurable passive microwave components. A rectangular patch antenna operating at $8 \mathrm{GHz}$ was designed and fabricated using inkjet printing, as shown in Figure 8 (a). The frequency of operation is almost twice the value of $f_{m}$, thus avoiding the lossy spectrum of the substrate. The antenna 


\section{WILEY-VCH}

has dimensions of $6.4 \times 7 \mathrm{~mm}$. The antenna was initially measured for its impedance properties without any magnetic bias, as shown in Figure 8 (b). Consequently, the antenna was characterized for its 3D radiation pattern, as shown in Figure 8 (c). The maximum gain of the antenna is approximately $-0.7 \mathrm{dBi}$ at $8.2 \mathrm{GHz}$. The radiation pattern of the antenna shows directional properties with maximum gain in the bore-sight direction as expected with a patch antenna. To test the tuning capability, the impedance of the antenna was measured in the presence of a magnetostatic field which was generated by an electromagnet. The strength of the applied magnetic field varied from 0 Oe to $5 \mathrm{kOe}$. No change in the resonant frequency of the antenna was observed up to a bias strength of $2 \mathrm{kOe}$ because the magnetic fields were lost in the air due to the demagnetization effect ${ }^{28}$. Above $2 \mathrm{kOe}$, the frequency of the antenna began to tune down as shown in Figure 8 (d). Increasing the fields beyond this value reduced the center frequency to $3.7 \mathrm{kOe}$. A total tuning range of $1.25 \mathrm{GHz}$ was obtained, which is 12.5 $\%$ of the center frequency. Further increasing the bias resulted in a slight increase in the resonant frequency of the antenna, which could be because the substrate was saturated for a bias field strength of $3.7 \mathrm{kOe}$. After this value, strong fields are required to tune the antenna using the Polder's equations ${ }^{30}$. The measured reflection coefficient of the antenna at a bias value of $3.7 \mathrm{kOe}$ is shown in Figure $8(\mathrm{~b})$. The antenna maintained its matching condition during the entire tuning range, which is required from such a design. No significant effect was expected on the antenna radiation pattern due to the applied bias, as it has been reported that the radiation pattern of a ferrite-based patch antenna does not change significantly in the biased state. ${ }^{[52]}$

\section{Conclusions}

In conclusion, this study successfully performed the preparation of the iron oxide nanoparticles and their ink-formulation to demonstrate the fully printed highly tunable inductor. Further, oleic acid functionalization and integration of nanoparticles with SU8 is 


\section{WILEY-VCH}

performed to fabricate the first printed free-standing magnetic substrate. The materials are characterized in detail to obtain the morphological, structural, chemical, optical, and magnetic properties. Furthermore, the printed substrate is characterized for its magnetostatic and microwave properties. The magnetic substrate demonstrates a saturation magnetization of 1560 Gauss and a calculated magnetization frequency of $4.37 \mathrm{GHz}$. To prove the functionality of the ink, a patch antenna design was implemented. The antenna successfully demonstrates the frequency tuning due to the application of magnetostatic fields across it. For a center frequency of $8 \mathrm{GHz}$, a tuning range of $12.5 \%$ was achieved at a magnetic field strength of 3.7 kOe. Such a functional ink is not only highly suitable for tunable and reconfigurable microwave devices, but could also be explored in sensing, biotechnology, and biomedical areas.

\section{Experimental Section}

Chemicals: Iron (II) chloride tetrahydrate $\left(\mathrm{FeCl}_{2} \cdot 4 \mathrm{H}_{2} \mathrm{O}\right.$, reagent plus, $\left.98 \%\right)$, iron (III) chloride hexahydrate $\left(\mathrm{FeCl}_{3} \cdot 6 \mathrm{H}_{2} \mathrm{O}, \mathrm{ACS}, 97-102 \%\right)$, sodium hydroxide ( $\mathrm{NaOH}$, Sigma Aldrich), acetic acid $\left(\mathrm{CH}_{3} \mathrm{COOH}\right.$, ACS reagent, $\left.\geq 99.7 \%\right)$, oleic acid $\left[\mathrm{CH}_{3}\left(\mathrm{CH}_{2}\right)_{7} \mathrm{CH}=\mathrm{CH}\left(\mathrm{CH}_{2}\right)_{7} \mathrm{COOH}\right.$, technical grade, 90\%], SU8 2002 (MicroChem), and ethanol (absolute, VWR Chemicals) were used as they were received, without further purification.

Synthesis of Iron Oxide NPs: As in a typical synthesis process, $0.01 \mathrm{M}$ iron (ii) chloride $(\sim 0.596 \mathrm{~g})$ and $0.02 \mathrm{M}$ iron (iii) chloride $(\sim 1.621 \mathrm{~g})$ were dissolved in $300 \mathrm{~mL}$ of deionized (DI) water, followed by mixing of $1 \mathrm{~mL}$ of acetic acid. The resulting solution was then slowly heated in a three-necked refluxing pot while stirring $(1000 \mathrm{rpm})$. When the temperature reached $90{ }^{\circ} \mathrm{C}, 2 \mathrm{~g}$ of $\mathrm{NaOH}$ was added. This resulted in a black solution, indicating the formation of $\mathrm{Fe}_{3} \mathrm{O}_{4}$ NPs. In this reaction condition, sodium hydroxide acts as a basic source and acetic acid to break the precipitates for the formation of uniform and disperse $\mathrm{Fe}_{3} \mathrm{O}_{4} \mathrm{NPs}$. 


\section{WILEY-VCH}

After 10 to15 minutes of refluxing, the black colloidal solution was obtained followed by centrifugation at $3000 \mathrm{rpm}$ for two minutes, and washing with deionized water and ethanol. Ink Formulation and Inkjet Printing Using Iron Oxide NPs: The as-prepared iron oxide NPs were formulated as ink in $3 \mathrm{~mL}$ of de-ionized water. Initially, the ink exhibited a high surface tension (SFT) of $\sim 63 \mathrm{mN} / \mathrm{m}$ which was adjusted with the addition of 10 volume percent of ethanol. After the addition of ethanol, ink exhibited an SFT of $\sim 44 \mathrm{mN} / \mathrm{m}$, which is good for stable jetting performance, as shown in supporting information Figure S11. The resulting solution was then stirred for $24 \mathrm{~h}$. Subsequently, the formulated iron oxide ink was filtered by $0.45 \mu \mathrm{m}$ polypropylene (PP) whatman paper before jetting. The observed viscosity of asformulated ink for ink jet printing was $\sim 1.74 \mathrm{cP}$, using a spindle speed of $100 \mathrm{rpm}$ and shear rate of $132 \mathrm{~s}^{-1}$ at room temperature. However, the SU8 embedded iron oxide nanoparticles was showing the viscosity of $37.8 \mathrm{cP}$, using a spindle speed of $100 \mathrm{rpm}$ and shear rate of 132 $\mathrm{s}^{-1}$ at room temperature. The iron oxide dot and line patterns were directly printed on glass substrate using a drop-on-demand piezoelectric ink-jet nozzle (manufactured by Dimatix) with a diameter of $16 \mu \mathrm{m}$; the drop volume was $10 \mathrm{pL}$. The uniform and continuous ejection of droplets was achieved by adjusting various wave forms while applying a firing voltage of 33.2 $\mathrm{V}$ at a $5-\mathrm{kHz}$ printer velocity, as shown in supporting information Figure S12. The jetting velocity of ejected droplets was $\sim 3.3 \mathrm{~m} / \mathrm{s}$ and the cartridge print height was $\sim 0.3 \mathrm{~mm}$. The thickness of as-printed lines was varied by the number of overprinting layers using $40 \mu \mathrm{m}$ drop spacing.

Functionalization of Iron Oxide NPs and Their Ink Formulation: For functionalization, the wet-form of iron oxide NPs were dispersed in $50 \mathrm{~mL}$ of ethanol, followed by the addition of $0.2 \mathrm{~mL}$ of oleic acid. The resulting solution was then stirred for $24 \mathrm{~h}$ to ensure the physical absorption of oleic acid molecules on surfaces of iron oxide NPs. After stirring, the resulting solution was centrifuged at $4000 \mathrm{rpm}$ for 2 minutes and washed with ethanol 2-3 times per 


\section{WILEY-VCH}

cycle to remove access oleic acid molecules. The resulting functionalized iron oxide NPs were then ready to mix with the SU8 2002 solution.

Fabrication of a Printed Antenna on Magnetic Substrate: An in-house SOC ink was utilized in this work to print an antenna on magnetic substrate. The SOC ink can produce smooth and dense films; it is stable and transparent (full details are covered in the reference). ${ }^{[15,20]}$ The antenna was printed on magnetic substrate $(t=\sim 1500 \mu \mathrm{m})$ using eight layers of AOC ink at 30 um drop spacing with a 10pL Dimatix DMP 2831 inkjet printer. A low-cost 250-watt IR lamp was used to cure the ink by placing the substrate under the lamp for five minutes after each printed layer. The maximum measured temperature of the substrate was $80^{\circ} \mathrm{C}$.

Characterization: The structural properties were examined using scanning electron microscopy (Zeiss Merlin with Gemini 2 column) and transmission electron microscopy (FEI Titan G2 80-300 kV equipped with a 2 k $\times 2$ k CCD camera model US4000, Gatan, Inc). The elemental quantification was examined with energy dispersive spectroscopy (EDS) equipped with FEI Nova Nano. In addition, the thicknesses and uniformity of printed features on substrates were measured using a surface profiler (Veeco Dektak 150). The crystallinity of the iron oxide powders was examined by X-ray diffraction (Bruker D8 Advance) in the range of 20 to $70^{\circ}$ at $40 \mathrm{kV}$. Furthermore, the UV-Vis absorption spectrum of the ink was obtained using a UV-Vis spectrophotometer (Cary 100 UV-Vis-NIR) with a standard $1 \mathrm{~cm}$ liquid cuvette and a background calibration that was run using ethanol. The chemical functionalization has been characterized by FT-IR spectrometers (Nicolet 6700). The FTIR sample is prepared using $\mathrm{KBr}$ pellet method. Approximately 0.1 to $1.0 \%$ sample is well mixed into 200 mg fine $\mathrm{KBr}$ powder and then finely pulverized using stone mortar and pestle. After pulverization, the resultant powder is placed in to a pellet-forming die for making transparent pellets. In order to correct the infrared light scattering loses in the pellet, a background measurement was done on a pellet holder with a pellet of $\mathrm{KBr}$ only. Finally, the sample was loaded and its measured infrared spectrum was recorded. Furthermore, viscosities 


\section{WILEY-VCH}

of the inks were measured using a Brookfield Rheometer (DV3T). The surface tensions of the inks were measured by using a KRUSS DSA100 based on pendant drop method. The particle size analysis of the ink was done using Zetasizer (Malvern instrument). Before analysis, ink was diluted 10-times with de-ionized (DI) water. The magnetic properties of iron oxide nanoparticles were examined by SQUID-VSM.

\section{Supporting Information}

Supporting Information is available from the Wiley Online Library or from the author.

\section{Acknowledgements}

The authors Dr. Mohammad Vaseem and Dr. Farhan Abdul Ghaffar contributed equally to this work. We acknowledge financial support from King Abdullah University of Science and Technology (KAUST) Office of Sponsored Research (OSR). We also would like to thank Nini Wei, Shuai Yang, Dr. Alessandro Genovese, Dr. Rachid Sougrat and Dr. Venkatesh Singaravelu for their work and assistance with SEM, TEM and magnetic analyses of the powder sample and films.

Received: ((will be filled in by the editorial staff))

Revised: ((will be filled in by the editorial staff)) Published online: ((will be filled in by the editorial staff))

\section{References}

[1] J.-H. Choi, H. Wang, S. J. Oh, T. Paik, P. S. Jo, J. Sung, X. Ye, T. Zhao, B. T. Diroll, C. B. Murray, C. R. Kagan, Science 2016, 352, 205.

[2] M. Vaseem, A.-R. Hong, R.-T. Kim, Y.-B. Hahn, J. Mater. Chem. C 2013, 1, 2112.

[3] T. T. Baby, S. K. Garlapati, S. Dehm, M. Häming, R. Kruk, H. Hahn, S. Dasgupta, ACS Nano 2015, 9, 3075.

[4] W. J. Scheideler, R. Kumar, A. R. Zeumault, V. Subramanian, Adv. Funct. Mater. 2017, $27,1606062$.

[5] S.-I. Park, Y. Xiong, R.-H. Kim, P. Elvikis, M. Meitl, D.-H. Kim, J. Wu, J. Yoon, C.-J. Yu, Z. Liu, Y. Huang, K.-c. Hwang, P. Ferreira, X. Li, K. Choquette, J. A. Rogers, Science 2009, 325, 977. 


\section{WILEY-VCH}

[6] S. Jung, A. Sou, K. Banger, D.-H. Ko, P. C. Y. Chow, C. R. McNeill, H. Sirringhaus, Adv. Energy Mater. 2014, 4, 1400432.

[7] D.-H. Lien, Z.-K. Kao, T.-H. Huang, Y.-C. Liao, S.-C. Lee, Jr-Hau He, ACS Nano 2014, 8, 7613.

[8] B. Huber, P. B. Popp, M. Kaiser, A. Ruediger, and C. Schindler, Appl. Phys. Lett. 2017, $110,143503$.

[9] D. McManus, S. Vranic, F. Withers, V. Sanchez-Romaguera, M. Macucci, H. Yang, R. Sorrentino, K. Parvez, S.-K. Son, G. Iannaccone, K. Kostarelos, G. Fiori, C. Casiraghi, Nature Nanotechnology 2017

[10] T. Vuorinen, J. Niittynen, T. Kankkunen, T. M. Kraft, Matti Mäntysalo, Sci. Rep. 2016, 6, 35289.

[11] A. Quddious, S. Yang, M. M. Khan, F. A. Tahir, A. Shamim, K. N. Salama, H. M. Cheema, MDPI Sensors 2016, 16.

[12] M. F. Farooqui, K. N. Salama, A. Shamim, Adv. Mater. Technol. 2017, 1700051.

[13] R. D. Boehm, P. R. Miller, J. Daniels, S. Stafslien, R. J. Narayan, Materials Today 2014, 17,247

[14] S. V. Murphy, A. Atala, Nature Biotechnology 2014, 32, 773.

[15] M. Vaseem, G. McKerricher, A. Shamim, ACS Appl. Mater. \& Inter. 2015, 8, 177.

[16] M. F Farooqui and A. Shamim, Sci. Rep. 2016, 6, 28949.

[17] G. McKerricher, R. Maller, V. Mohammad, M. A. McLachlan, A. Shamim, Ceramics International 2017, 43, 9846.

[18] W. J. Hyun, E. B. Secor, G. A. Rojas, M. C. Hersam, L. F. Francis, C. D. Frisbie, Adv. Mater. 2015, 27, 7058.

[19] G. Grau, V. Subramanian, Adv. Electron. Mater. 2016, 2, 1500328.

[20] G. McKerricher, M. Vaseem, A. Shamim, Microsystems \& Nanoengineering 2017, 3, 16075 


\section{WILEY-VCH}

[21] N. Ning, X. P. Li, J. Fan, W. C. Ng, Y. P. Xu, X. Qian, H. L. Seet, IEEE Transactions on Magnetics 2006, 42, 1585

[22] L.-R. Tan, R.-X. Wu, C.-Y. Wang, Y. Poo, IEEE Antennas and Wireless Propagation Letters 2013, 12, 273.

[23] H. Lee, B. S. Cook, K. P. Murali, M. Raj, M. M. Tentzeris, IEEE Microwave and Wireless Components Letters, 2016, 26, 419.

[24] D. M. Pozar, V. Sanchez, Elect. Lett. 1988, 24, 729.

[25] K. K. Tsang, R. J. Langley, IEE Proc. Micro. Ant. Prop. 1998, 145, 49.

[26] E. Arabi, F. A. Ghaffar, A. Shamim, IEEE Micro. Wless. Comp. Lett. 2015, 25, 16.

[27] F. A. Ghaffar, J. Bray, A. Shamim, IEEE Trans. Ant. Prop. 2014, 62, 1238.

[28] F. A. Ghaffar, Atif Shamim, IEEE Ant. Wless Prop. Lett. 2015, 14, 831.

[29] F. A. Ghaffar, A. Shamim, IEEE Trans. Mag. 2015, 51, 1.

[30] A. Nafe, A. Shamim, IEEE Trans. Micro. Th. \& Tech. 2015, 63, 2264.

[31] H. Lee, M. M. Tentzeris, P. M. Raj, K. P. Murali, Y. Kawahara, Antennas and Propagation Society International Symposium (APSURSI), 2013 IEEE

[32] H. Song, J. Spencer, A. Jander, J. Nielsen, J. Stasiak, V. Kasperchik, P. Dhagat, J. Appl. Phys. 2014, 115, 17E308.

[33] M. Bissannagari, J. Kim, Ceramics International 2015, 41, 8023.

[34] http://www.sigmaaldrich.com/materials-science/material-scienceproducts.html?TablePage $=119470654$

[35] http://www.microchem.com/Prod-SU8_KMPR.htm

[36] K. Bhattacharya, P. Deb, Dalton Trans. 2015, 44, 9221.

[37] V. Patsula, L. Kosinová, M. Lovrić, L. F. Hamzić, M. Rabyk, R. Konefal, A. Paruzel, M. Šlouf, V. Herynek, S. Gajović, D. Horák, ACS Appl. Mater. Interfaces 2016, 8, 7238.

[38] T. Wu, Y. Liu, X. Zeng, T. Cui, Y. Zhao, Y. Li, G. Tong, ACS Appl. Mater. Interfaces 2016, 8,7370 . 


\section{WILEY-VCH}

[39] T.-I Yang, C.-Y. Chuang, S.-C. Yang, L. C. Kempel, P. Kofinas, Adv. Eng. Mater. 2016, 18,121

[40] W. Wu, Z. Wu, T. Yu, C. Jiang and W.-S. Kim, Sci. Technol. Adv. Mater. 2015, 16, 023501.

[41] J.-C. Si, Y. Xing, M.-L. Peng, C. Zhang, N. Buske, C. Chen, Y.-L. Cui, Cryst. Eng. Comm. 2014, 16, 512.

[42] T. Marín, P. Montoya, O. Arnache, J. Calderón, J. Phys. Chem. B 2016, 120, 6634.

[43] D.-Y. Kang, C. Kim, G. Park, J. H. Moon, Sci. Rep. 2015, 5, 18185.

[44] L. Zhuang, Y. Zhao, H. Zhong, J. Liang, J. Zhou, H. Shen, Sci. Rep. 2015, 5, 17063.

[45] F. N. Sayed, V. Polshettiwar, Sci. Rep. 2015, 5, 9733.

[46] W. Wu, Z. Wu, T. Yu, C. Jiang, W.-S. Kim, Sci. Technol. Adv. Mater. 2015, 16, 023501.

[47] M. Vaseem, N. Tripathy, G. Khang, Y.-B. Hahn, RSC Adv. 2013, 3, 9698.

[48] D. M. Pozar, IEEE Transactions on Antennas and Propagation, 1992, 40, 1084.

[49] A. Shamim, J. Bray, N. Hojjat, L. Roy, IEEE Trans. Adv. Packag. 2011, 1, 999.

[50] F. A. Ghaffar, M. Vaseem, M. F. Farooqui, A. Shamim, IEEE MTT-S International Microwave Symposium (IMS), 2016, pp. 1-3.

[51] J. R. Bray, K. T. Kautio, and L. Roy, IEEE Trans. Adv. Packag. 2004, 27, 558.

[52] A. Petosa, E. K. Mongia, M. Cuhaci and J. S. Wight, IEEE Electronic Lett. 1994, 30, 1021. 


\section{WILEY-VCH}

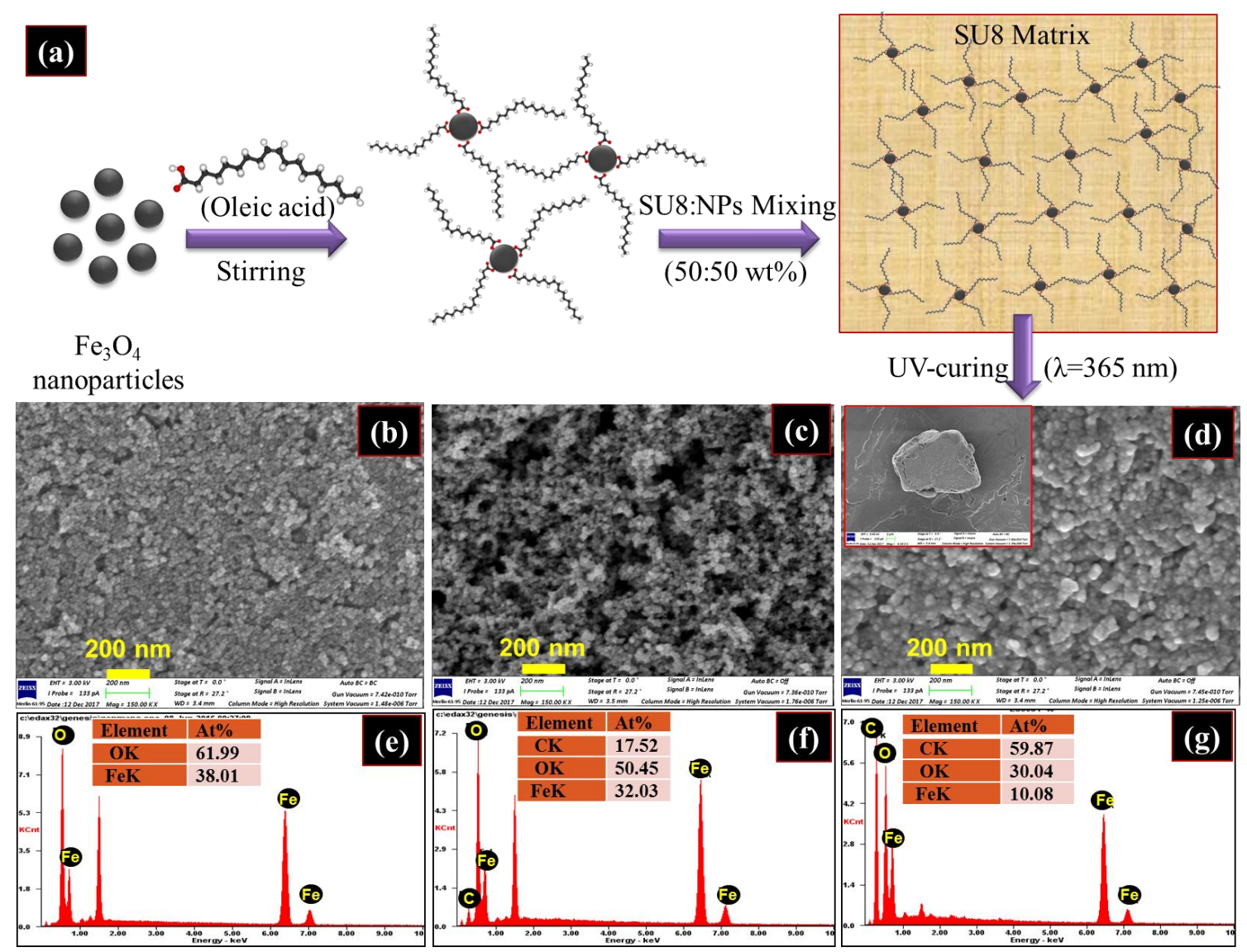

Figure 1. (a) An illustrative diagram of the functionalization of iron oxide nanoparticles and mixing with SU8. SEM and EDX analysis of (b, e) pure iron oxide, (c, f) oleic acid functionalized, and (d, g) SU8-mixed iron oxide nanoparticles. The inset in (d) is showing low resolution iron oxide nanoparticles embedded in SU8 matrix.
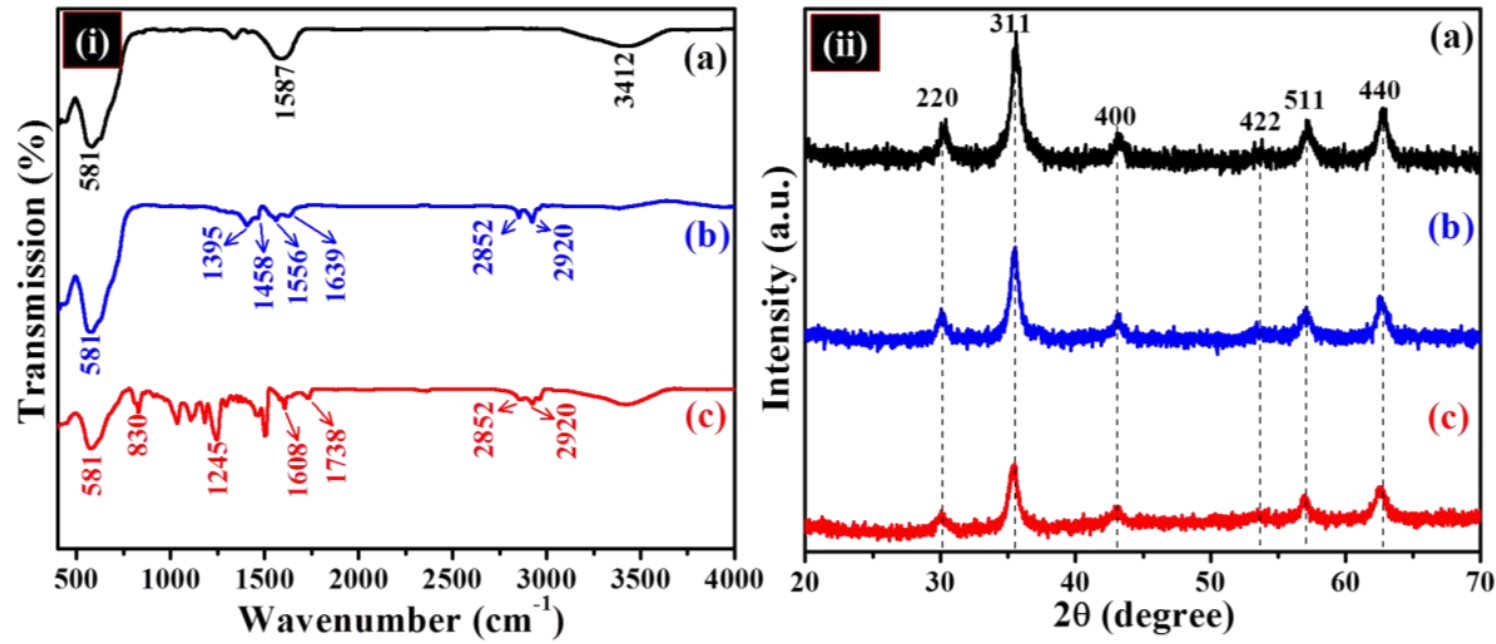

Figure 2. (i) FT-IR spectra and (ii) XRD patterns of (a) pure iron oxide, (b) oleic acid functionalized iron oxide nanoparticles, and (c) SU8-mixed iron oxide nanoparticles. 


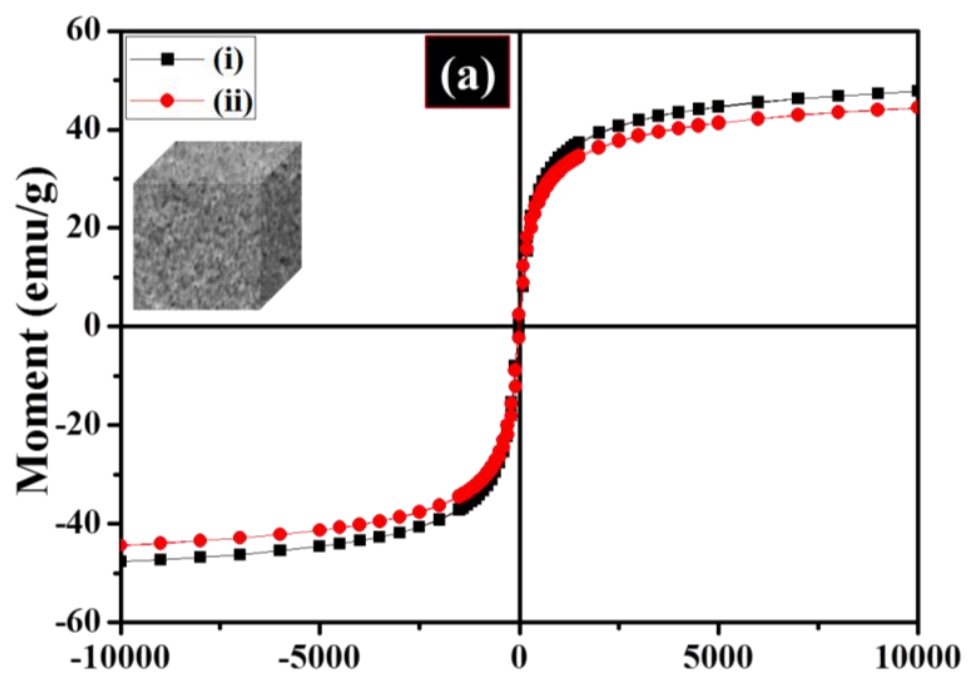

Magnetic Field (Oe)

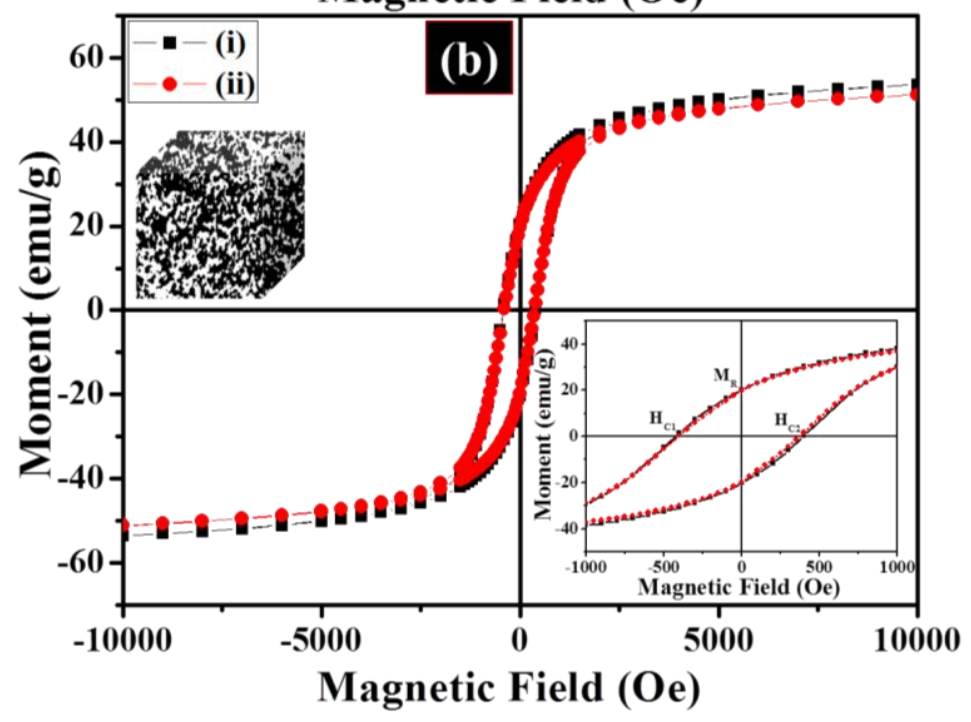

Figure 3. (a) Hysteresis loop of (i) pure iron oxide and (b) oleic acid functionalized iron oxide nanoparticles at (a) $300 \mathrm{~K}$ and (b) $5 \mathrm{~K}$, along with the full range of the hysteresis measured between -10000 and 10000 Oe (insert). The insets in (a) and (b) show the pictorial presentation of loosely bound nanoparticles in a freezing state. 


\section{WILEY-VCH}

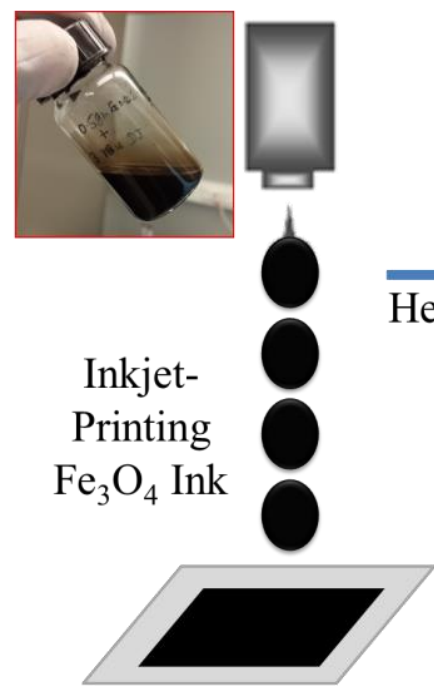

Printed Magnetic Film

(a)

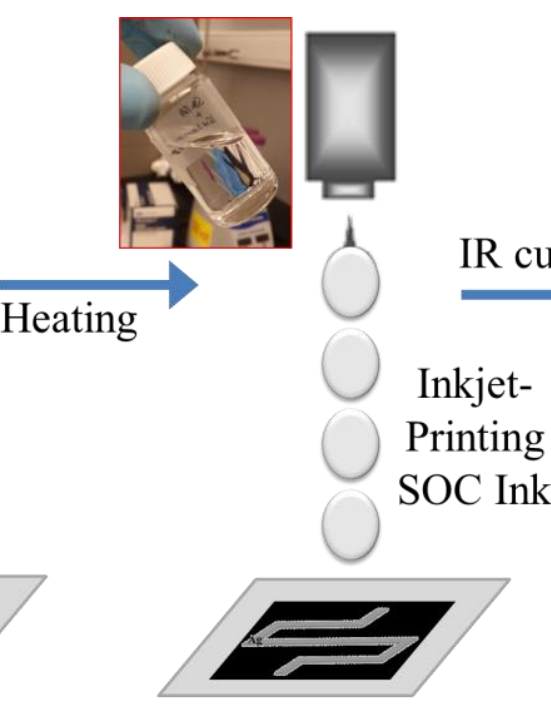

Printed Inductor

(b)

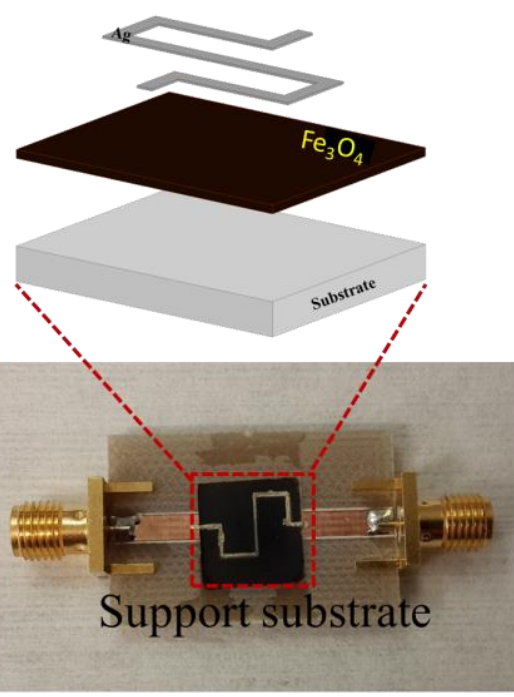

Tunable Inductor on magnetic

(c) film

Figure 4. Schematic presentation of the fabrication of fully printed tunable inductor
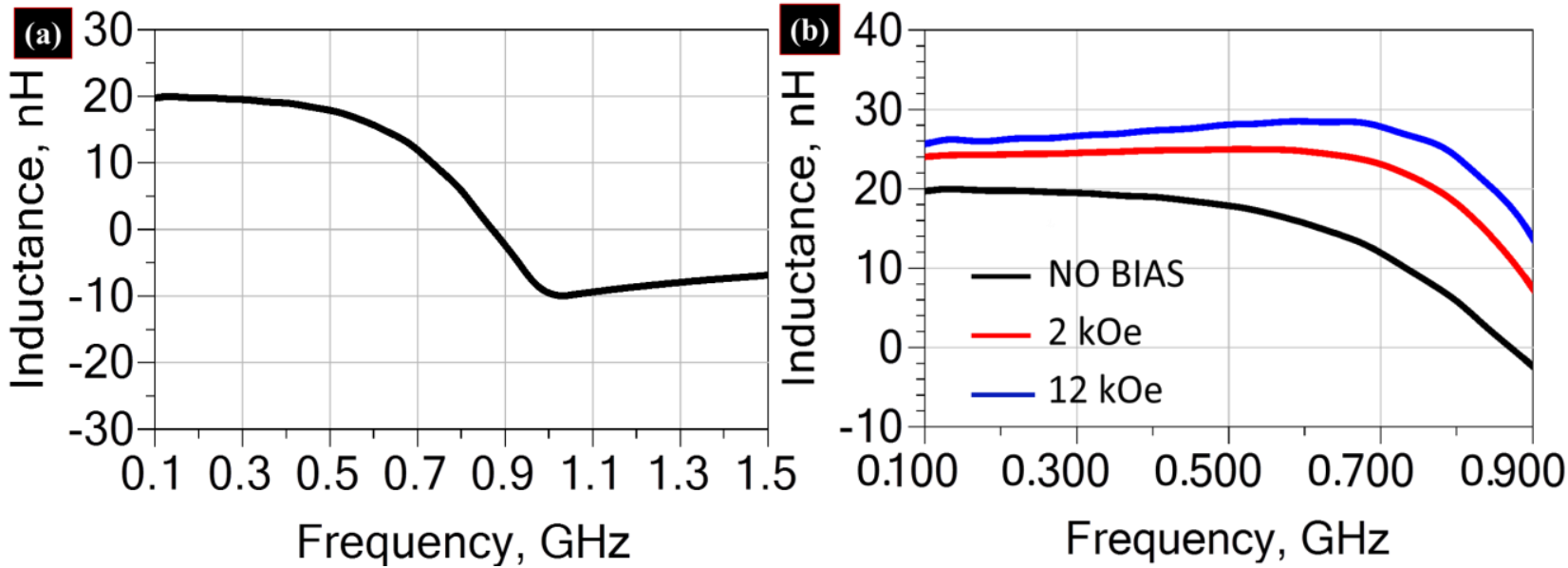

Figure 5. (a) Measured inductance showing the self-resonance frequency (SRF) and (b) zoom in view to show the change in inductance with magnetic field bias. 


\section{WILEY-VCH}

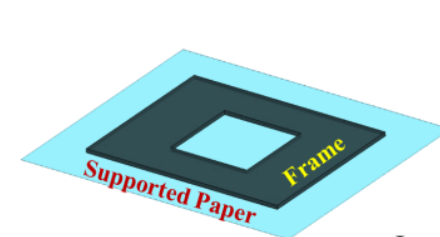

(a)

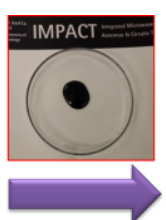

Iron oxide NPs:SU8 mixed ink printing

by squeegee

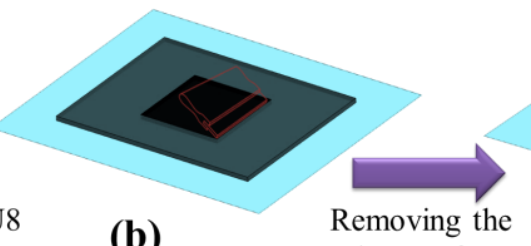
substrate from frame

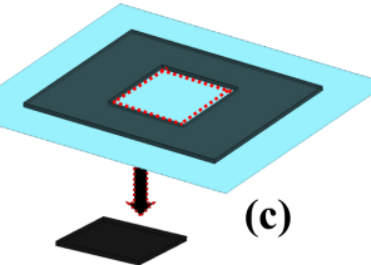

Free-standing Magnetic substrate

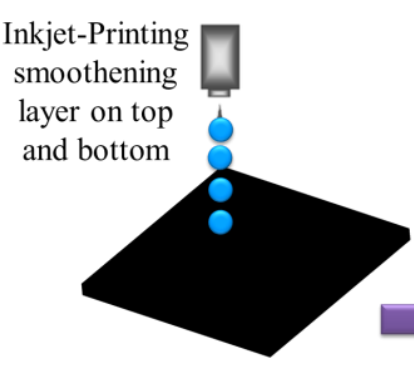

(d) Free-standing Magnetic substrate
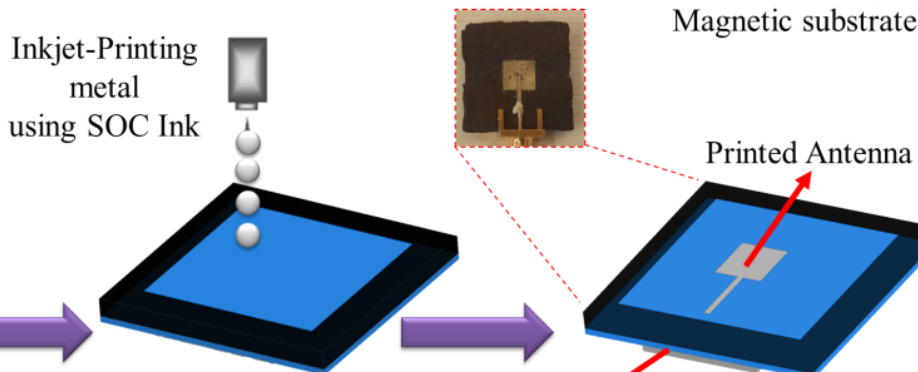

(e)

Printed metallic ground plane

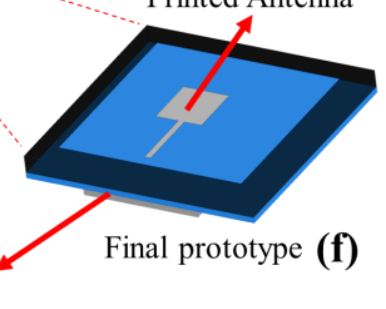

Figure 6. Schematic presentation of the fabrication of free-standing magnetic substrate followed by inkjet printing of a patch antenna.
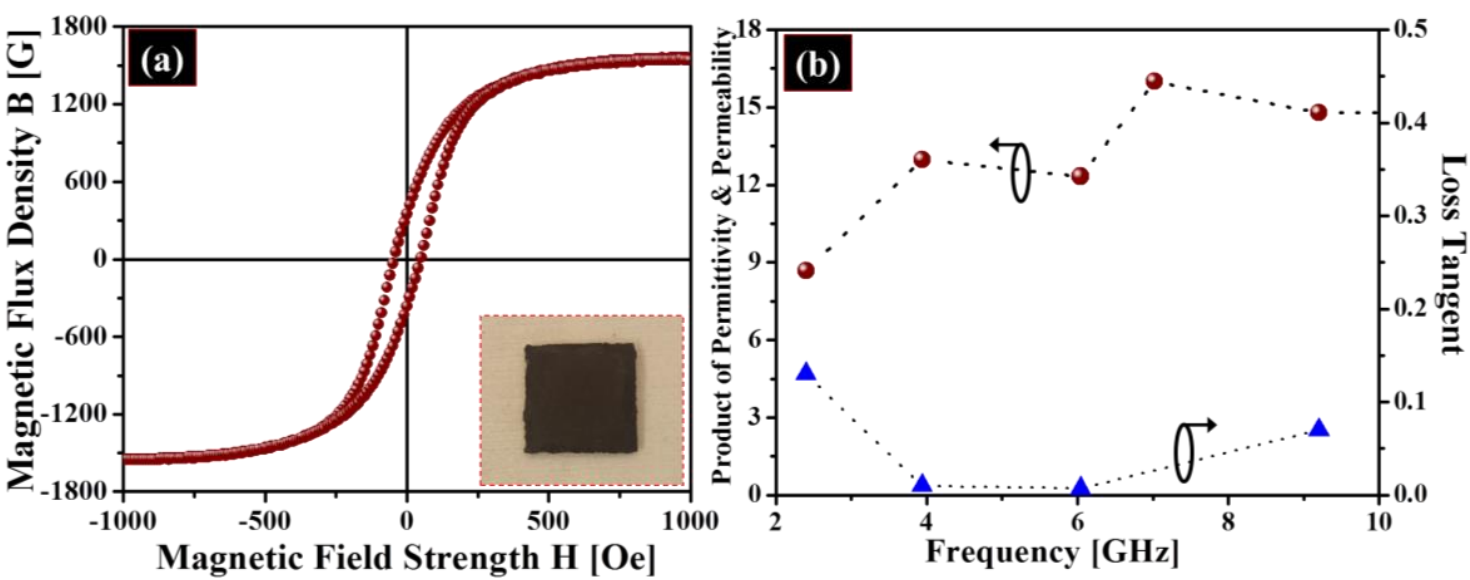

Figure 7. (a) Measured $\mathrm{B}(\mathrm{H})$ curve of printed free-standing substrate and (b) product of permittivity, and permeability and loss tangent of the magnetic ink vs frequency. 


\section{WILEY-VCH}
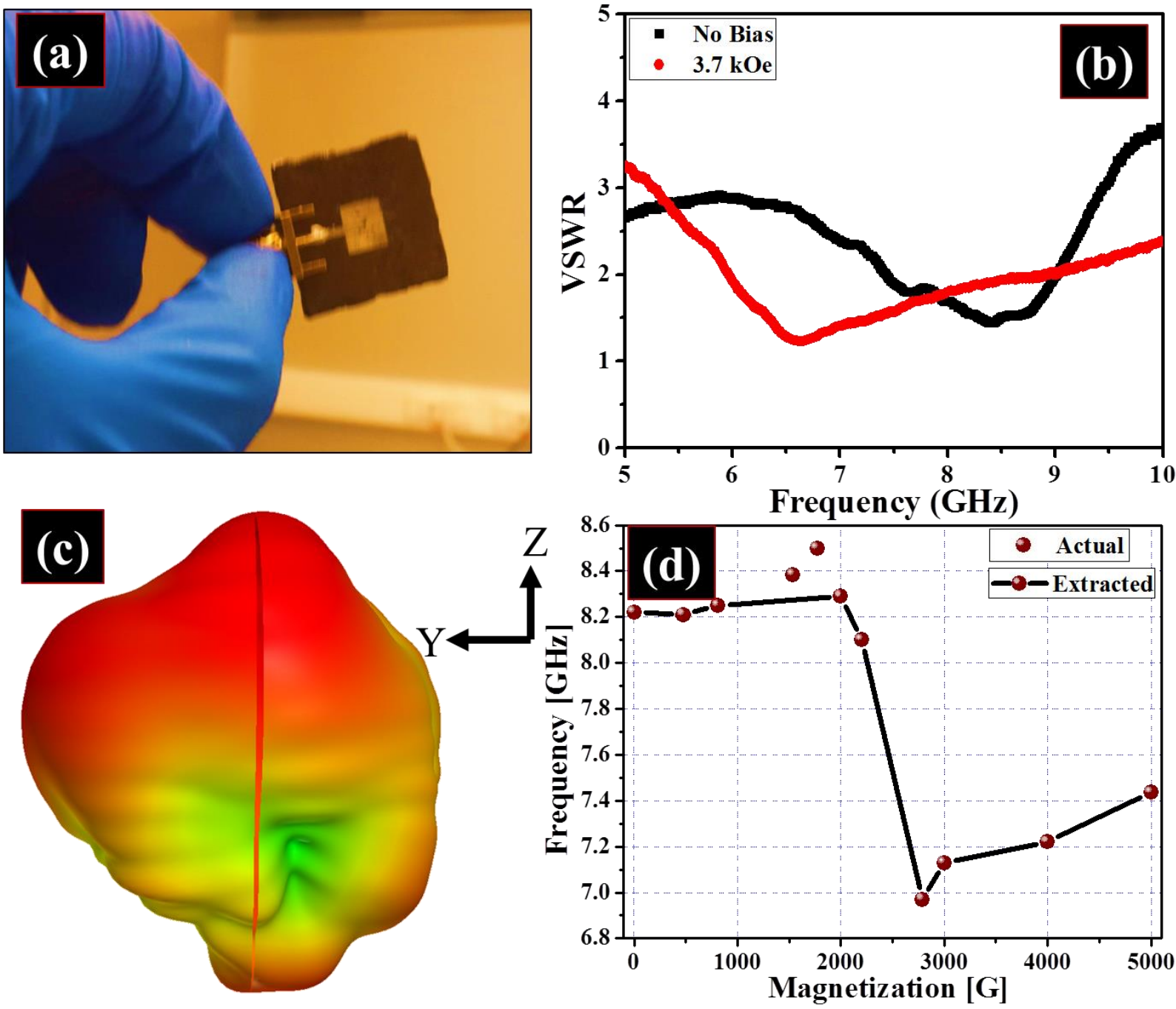

Figure 8. (a) Fabricated antenna on free-standing magnetic substrate, (b) measured frequency tuning, (c) measured 3D radiation pattern, and (d) S11 measurements for no bias and $3.7 \mathrm{kOe}$ bias for the inkjet-printed patch antenna.

Table 1. A performance comparison table for fully printed tunable inductor

\begin{tabular}{ccc}
\hline & $\begin{array}{c}\text { Saturated } \\
\text { Magnetization } \\
(\text { memu) }\end{array}$ & $\begin{array}{c}\text { Tunability (\%) } \\
\text { @ magnetic field }\end{array}$ \\
\hline $\begin{array}{c}\text { Our Iron Oxide } \\
\text { NPs ink } \\
\text { Commercial ink } \\
{[34]}\end{array}$ & 12.4 at $1 \mathrm{kOe}$ & $18 \%$ @ kOe \\
& 0.18 at $3 \mathrm{kOe}$ & $0.8 \%$ @ kOe \\
\hline
\end{tabular}


WILEY-VCH

Table 2. Permittivity and permeability of the magnetic ink at different frequencies

\begin{tabular}{ccc}
\hline $\begin{array}{c}\text { Frequency } \\
{[\mathrm{GHz}]}\end{array}$ & $\begin{array}{c}\text { Demagnetized } \\
\text { Permeability } \\
\left(\mu_{0}^{\prime}\right)\end{array}$ & $\begin{array}{c}\text { Permittivity } \\
\left(\varepsilon_{\text {eff }}\right)\end{array}$ \\
\hline 6.04 & 0.796 & 15.5 \\
7.9 & 0.86 & 15.29 \\
9.9 & 0.9 & 14.2 \\
\hline
\end{tabular}




\section{WILEY-VCH}

An iron oxide nanoparticle-based magnetic ink is developed, which has been completely characterized for its material properties, and then realized its utility through a fully printed, magnetically controllable RF device. This first demonstration of a fully printed RF device is an important milestone for the next generation of low-cost tunable and reconfigurable components that can be realized through additive manufacturing.

Iron Oxide Nanoparticle-based Ink, Inkjet Printing, Magnetic Properties, RF Characterization, Tunable Devices

M. Vaseem*, F. A. Ghaffar, M. F. Farooqui, A. Shamim

Iron Oxide Nanoparticle-Based Magnetic Ink Development for Fully Printed Tunable Radio-Frequency Devices

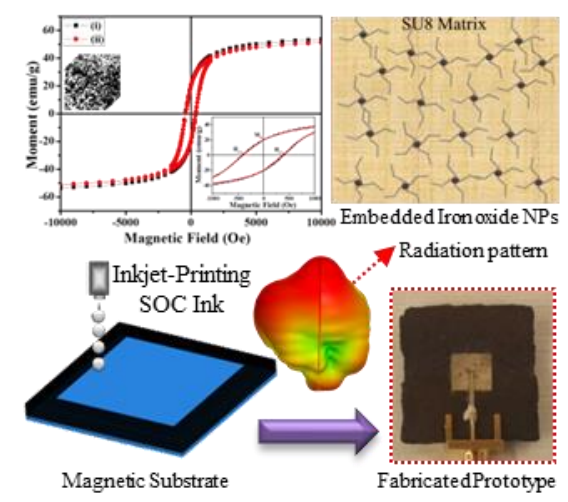




\section{WILEY-VCH}

((Supporting Information can be included here using this template))

Copyright WILEY-VCH Verlag GmbH \& Co. KGaA, 69469 Weinheim, Germany, 2016.

Supporting Information

Iron Oxide Nanoparticle-Based Magnetic Ink Development for Fully Printed Tunable Radio-Frequency Devices

Mohammad Vaseem*, Farhan Abdul Ghaffar, Muhammad Fahad Farooqui, and Atif Shamim
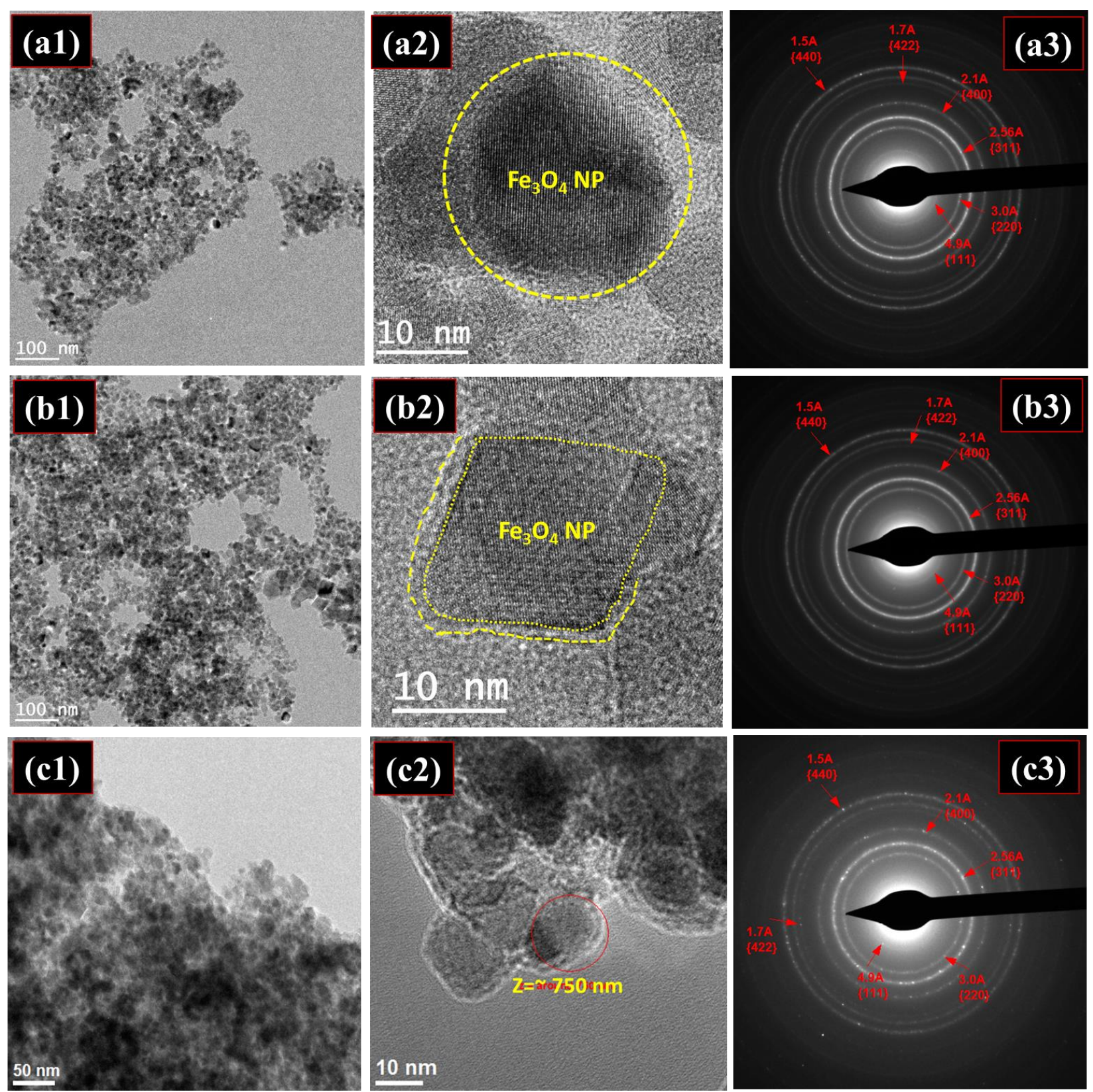

Figure S1. TEM, HR-TEM images, and SAED patterns of (a) pure iron oxide, (b) oleic acid functionalized, and (c) SU8-mixed iron oxide nanoparticles. 


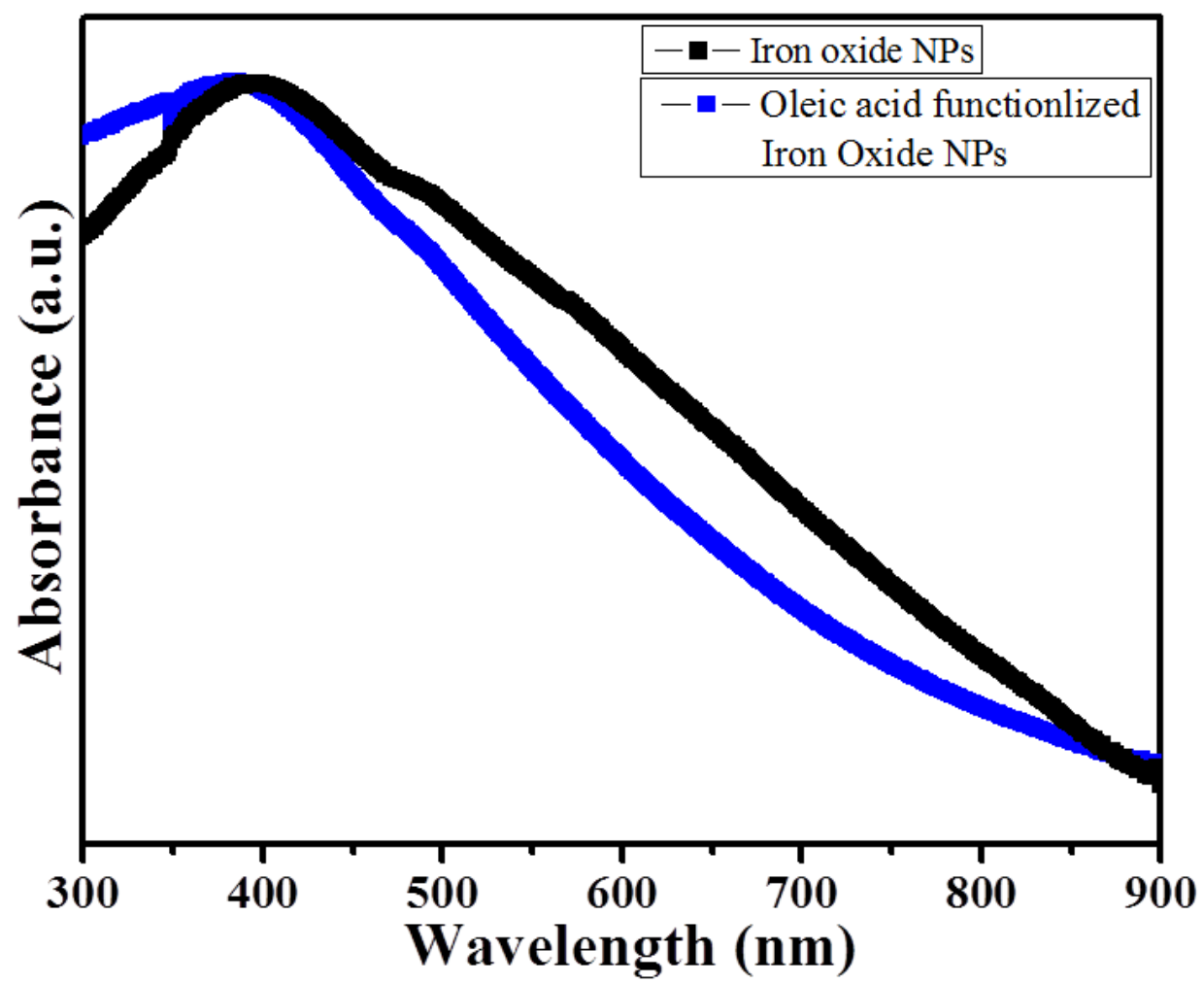

Figure S2. UV-Vis absorption spectra of pure and oleic acid functionalized iron oxide NPs.

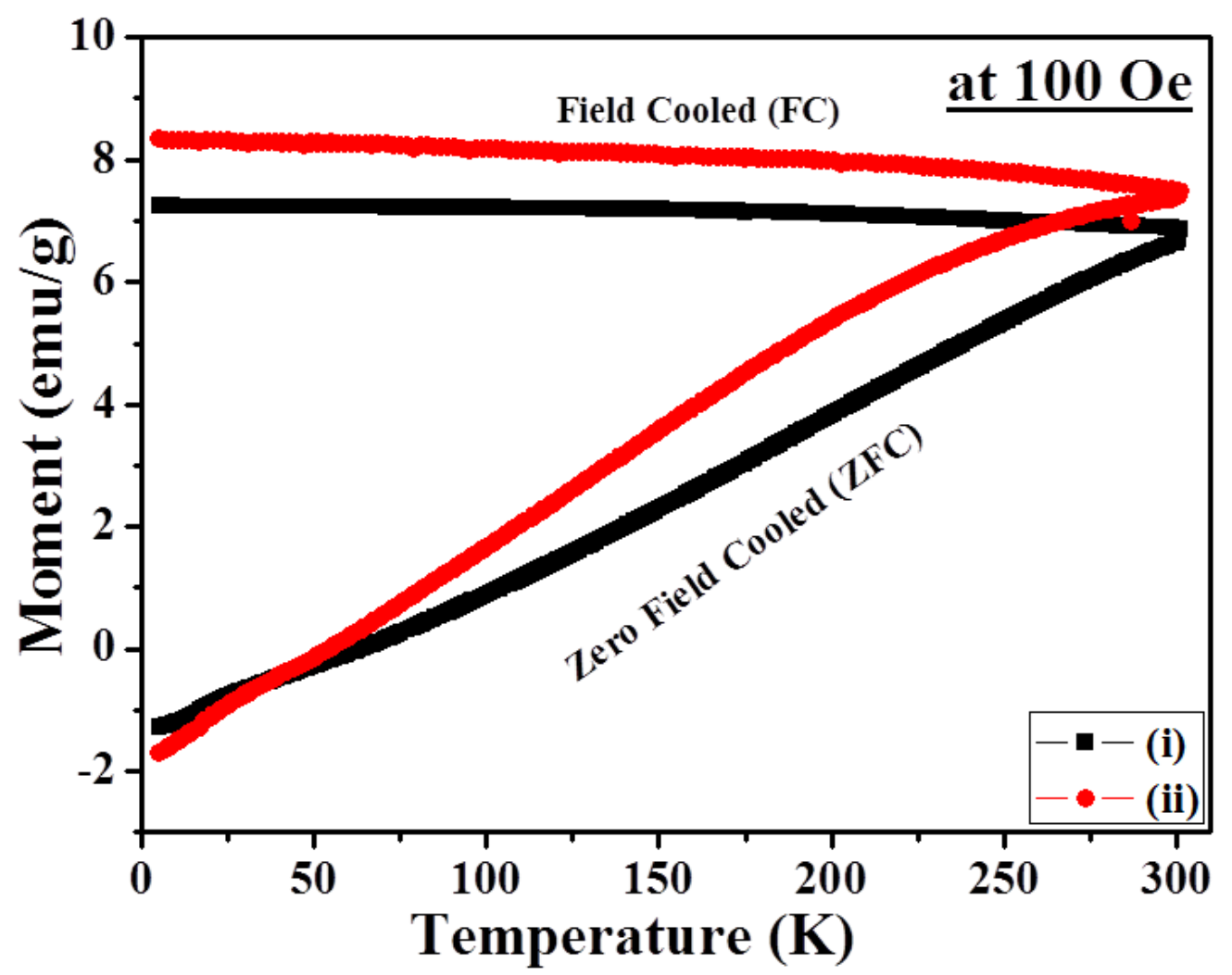

Figure S3. Temperature dependence magnetization of (i) pure and (ii) oleic acid functionalized iron oxide NPs in field cooled (FC) and zero field cooled (ZFC) at an applied field of 100 Oe. 


\section{WILEY-VCH}

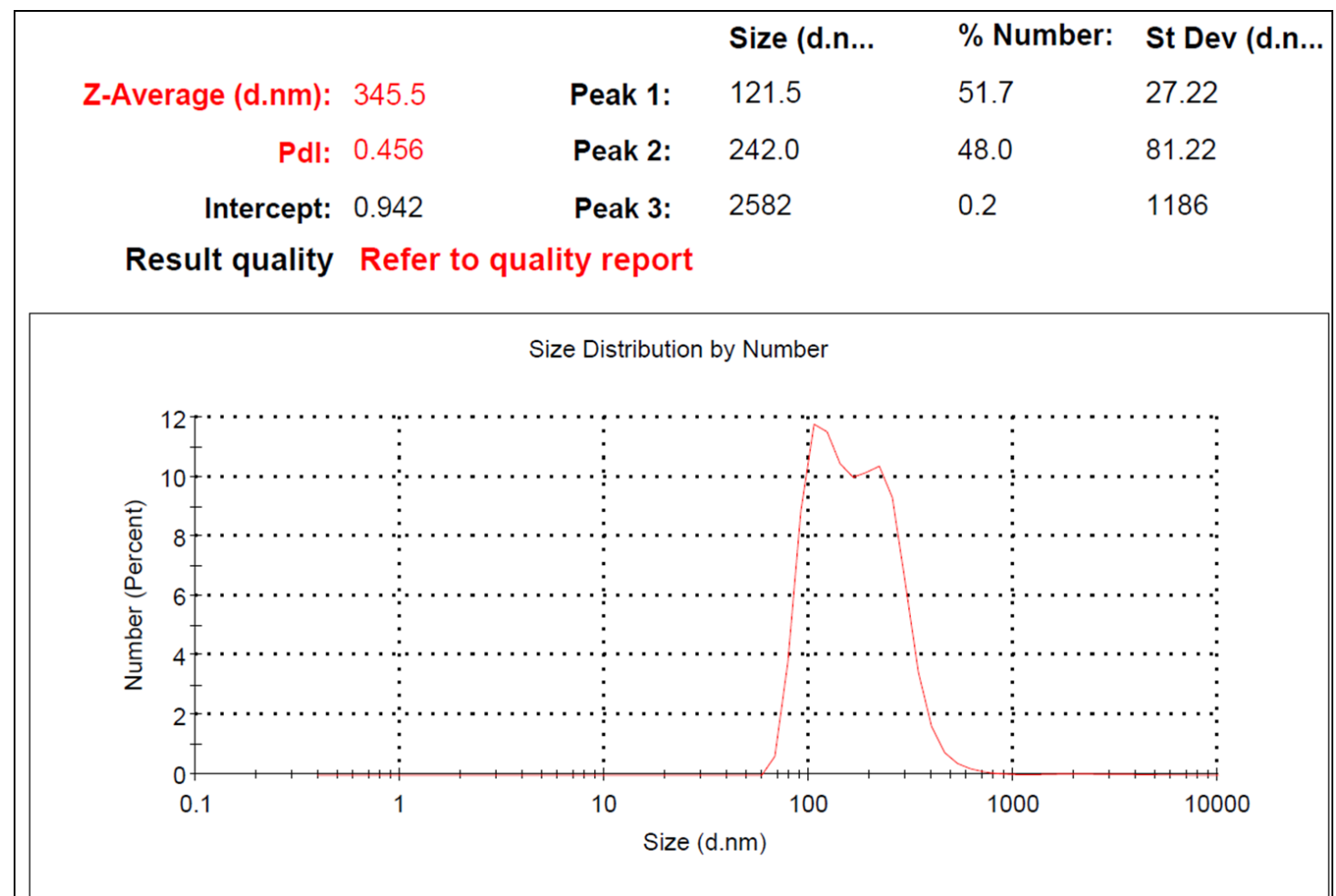

Figure S4. A graph showing particle size distribution. Before analysis, the as-formulated iron oxide nanoparticles ink was diluted 10-times with de-ionized (DI) water.
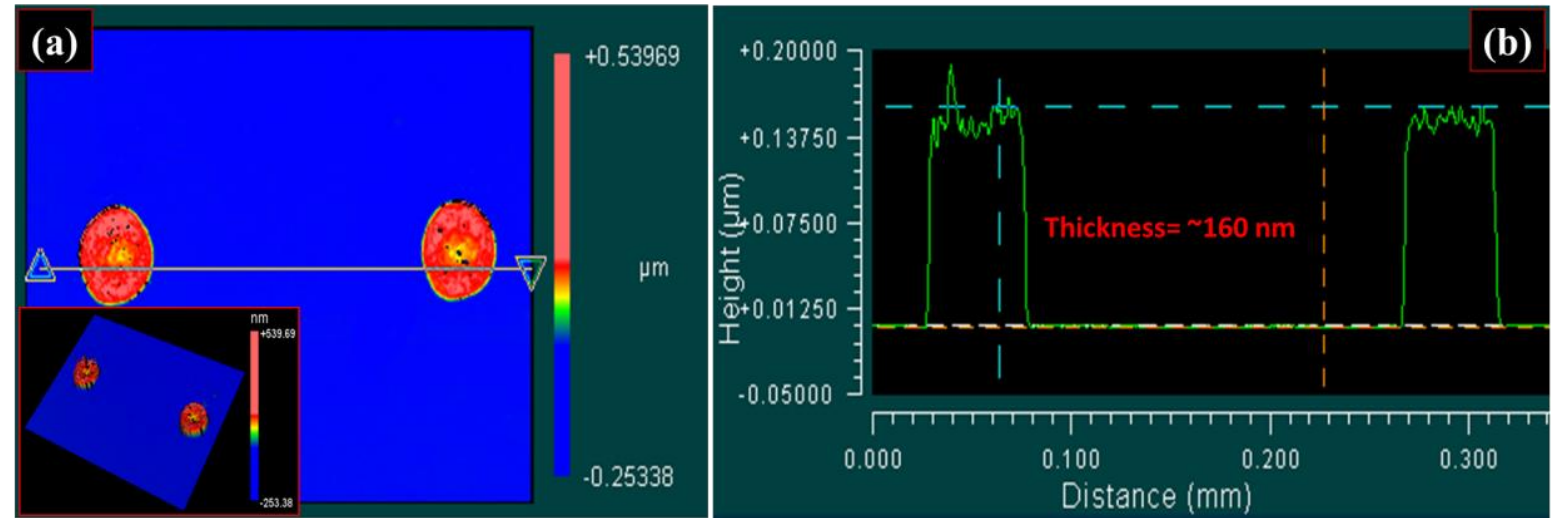

Figure S5. (a) 2D image of printed dots and its corresponding (b) surface profile. The inset in (a) shows the $3 \mathrm{D}$ view of the printed dots. 


\section{WILEY-VCH}
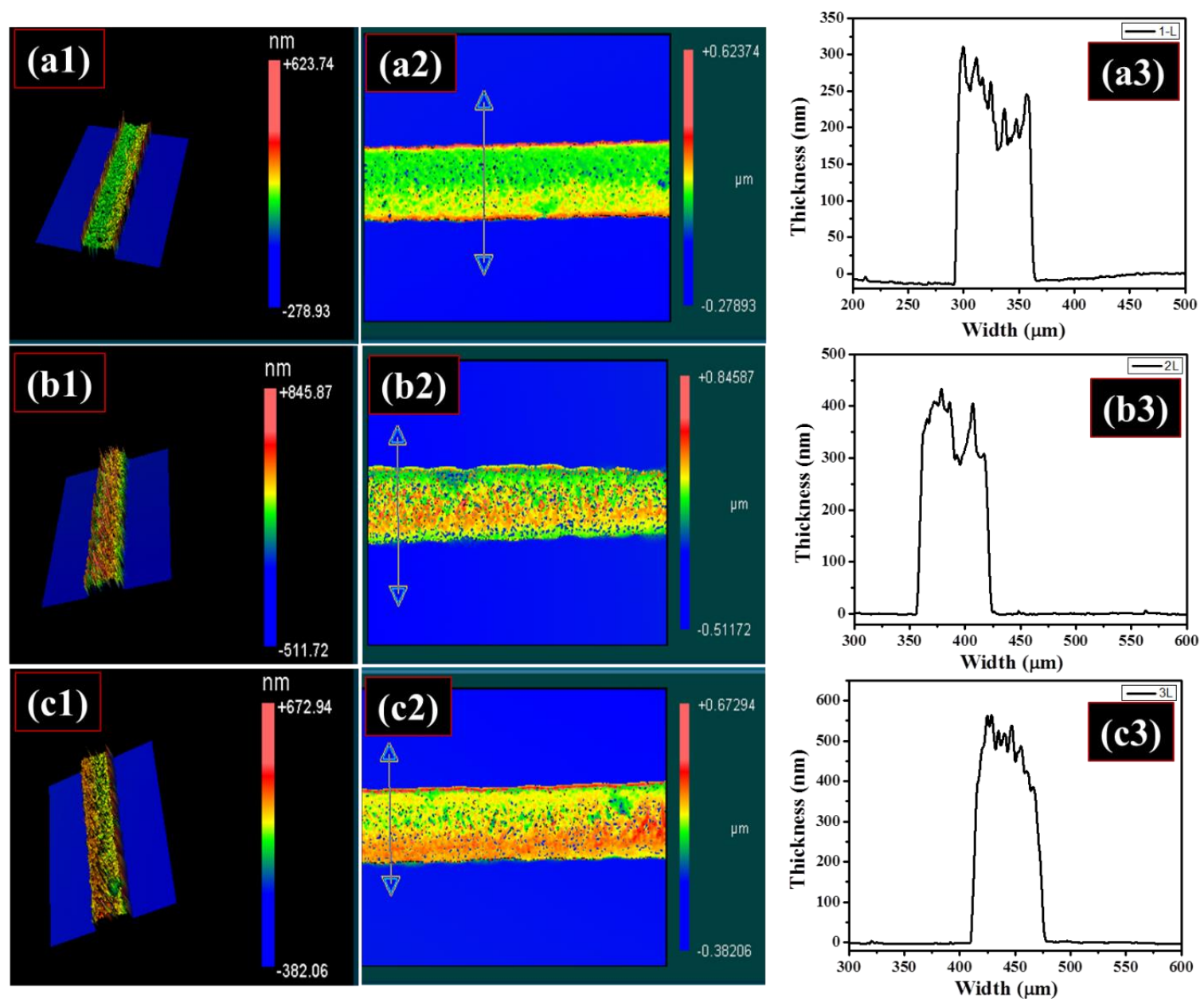

Figure S6. 3D, 2D and cross-sectional surface profiler images of inkjet-printed iron oxide: (a) single printing, (b) 2 over-layer, and (c) 3 over-layer. 


\section{WILEY-VCH}
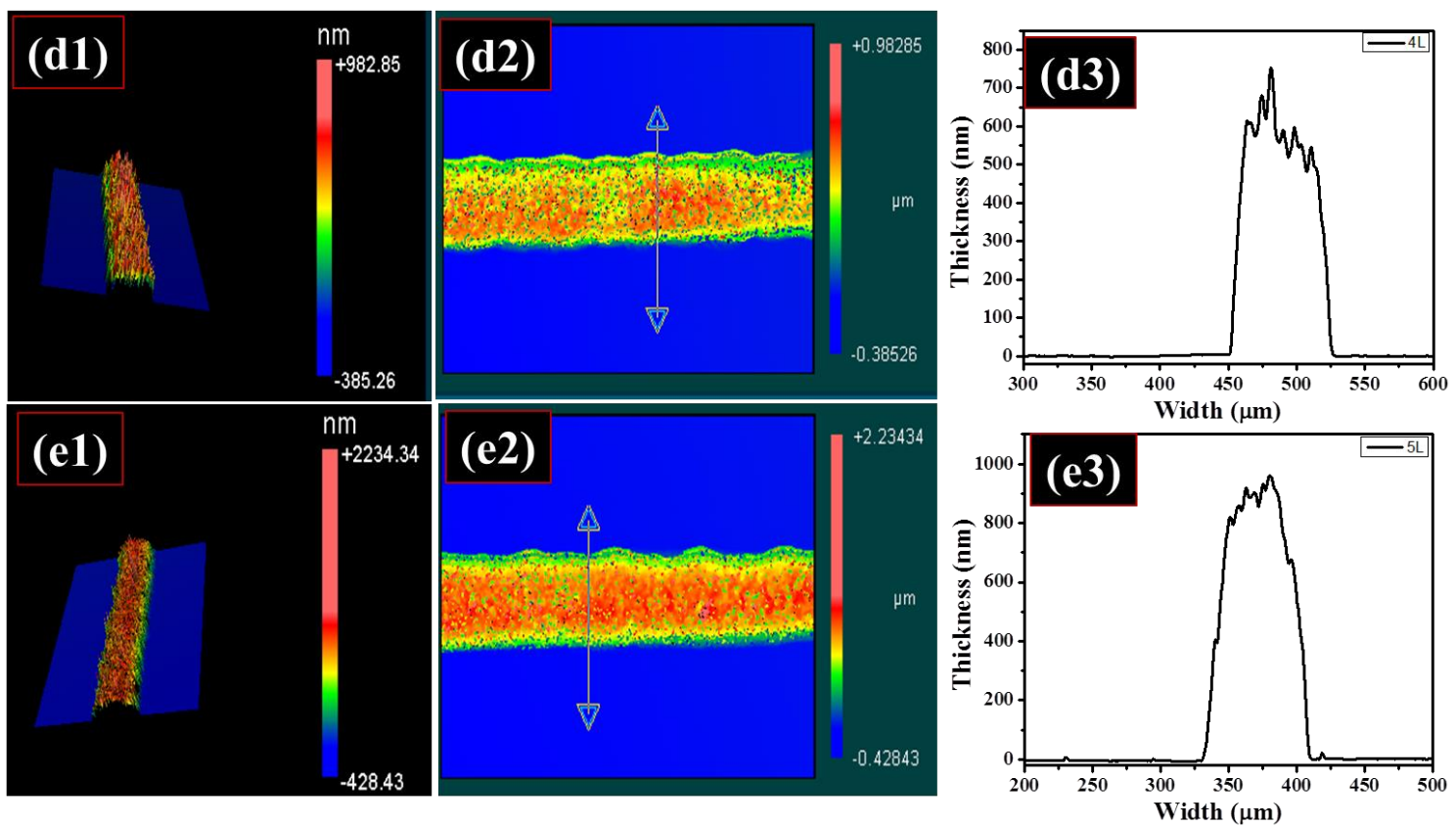

Figure S7. 3D, 2D and cross-sectional surface profiler images of inkjet-printed iron oxide: (d) 4 over-layer, and (c) 5 over-layer.

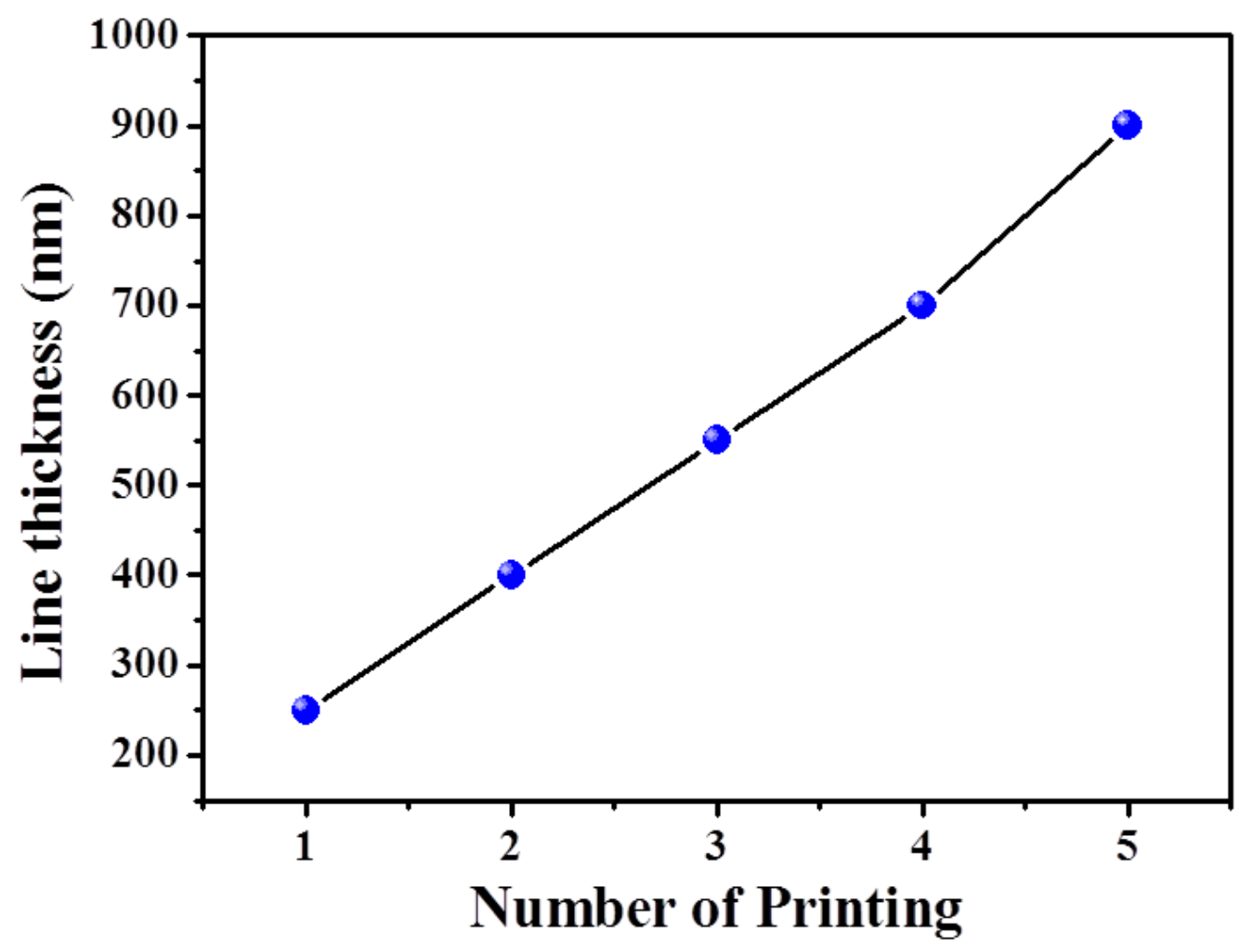

Figure S8. Graph showing thicknesses of inkjet-printed lines with number of printing. 


\section{WILEY-VCH}
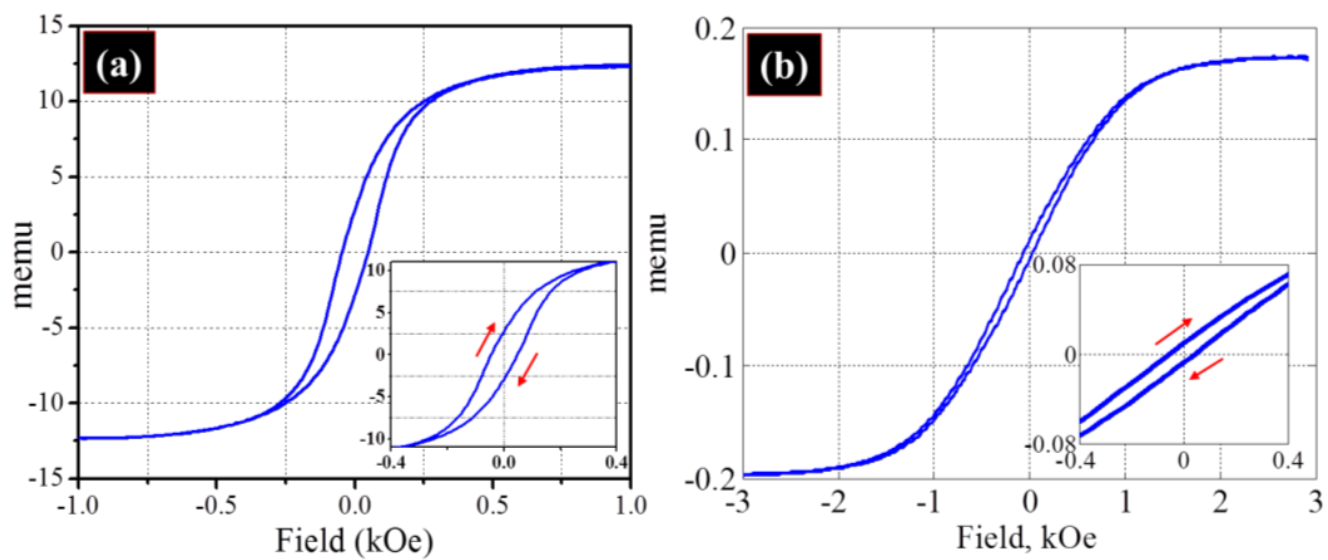

Figure S9. VSM measured hysteresis curve of (a) printed magnetic film using our iron oxide nanoparticles based ink and (b) using commercially available ink. The inset in (a) and (b) are showing zoomed hysteresis curve. Commercial ink shows the saturation magnetization of approximately 0.18 memu under applied field of $3 \mathrm{kOe}$ and the coercivity is found to be 51

Oe, as shown in (b).
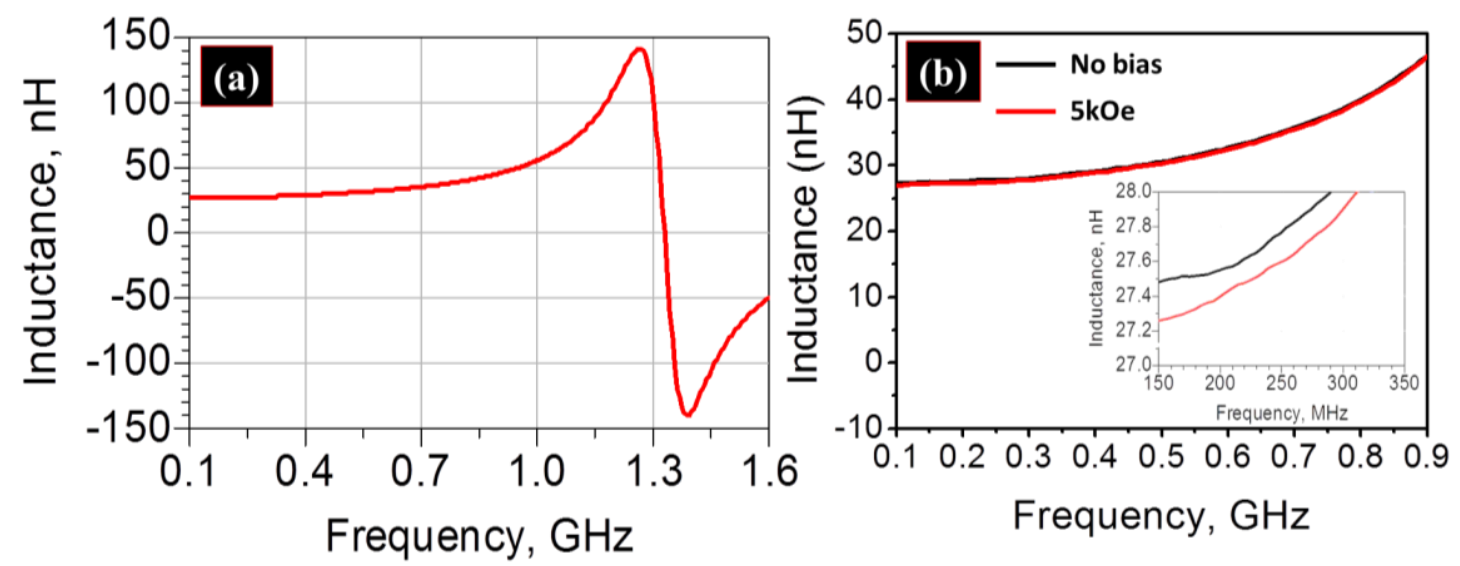

Figure S10. (a) Measured inductance for fully printed tunable inductor using commercial iron oxide ink and (b) change in inductance with magnetic field bias. The inset in (b) is showing zoom in view. In brief, commercial ink ${ }^{[34]}$ was printed with 10 layers with each layer having a thickness of around $100 \mathrm{~nm}$. The magnetic layer is then heated for 5 minutes at $160{ }^{\circ} \mathrm{C}$ to evaporate the solvent. The measured SRF of the inductor is $\sim 1.4 \mathrm{GHz}$ as shown in (a). 


\section{WILEY-VCH}
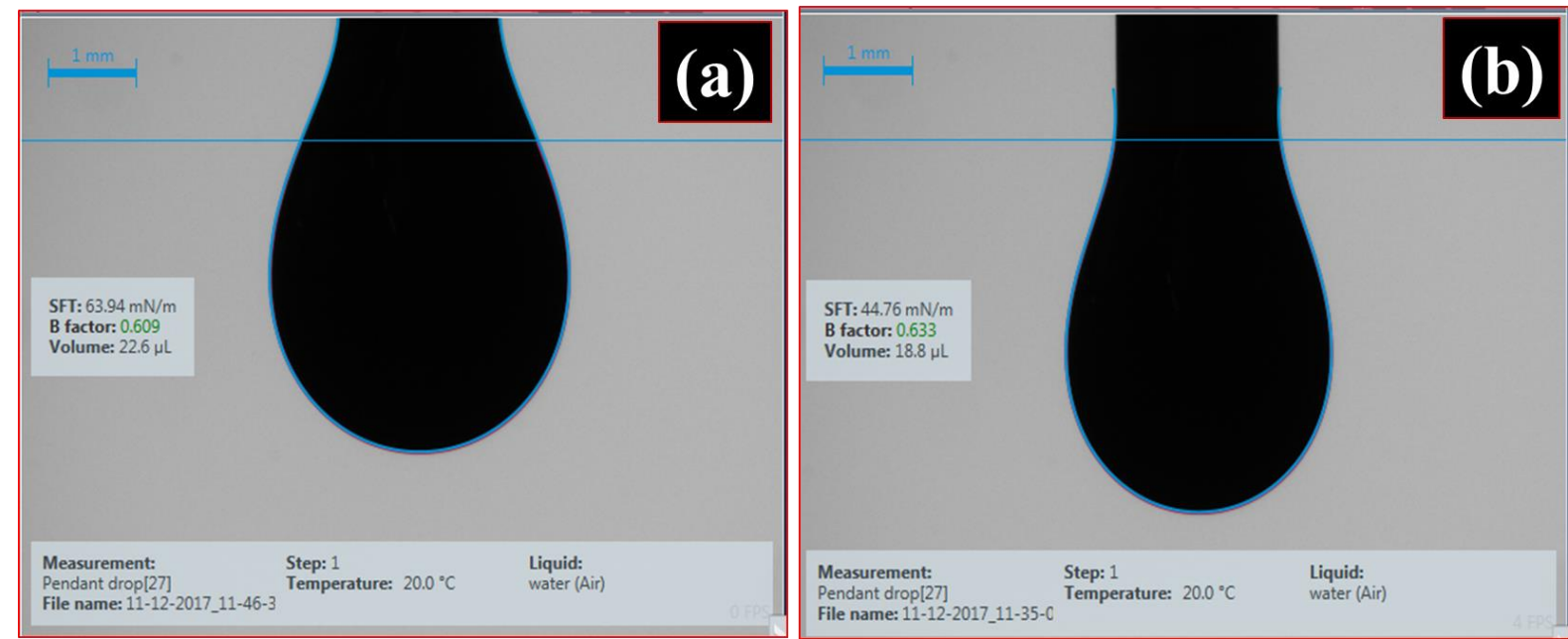

Figure S11. Surface tension (SFT) measurement of the ink (a) without ethanol and (b) with 10 vol \% ethanol.
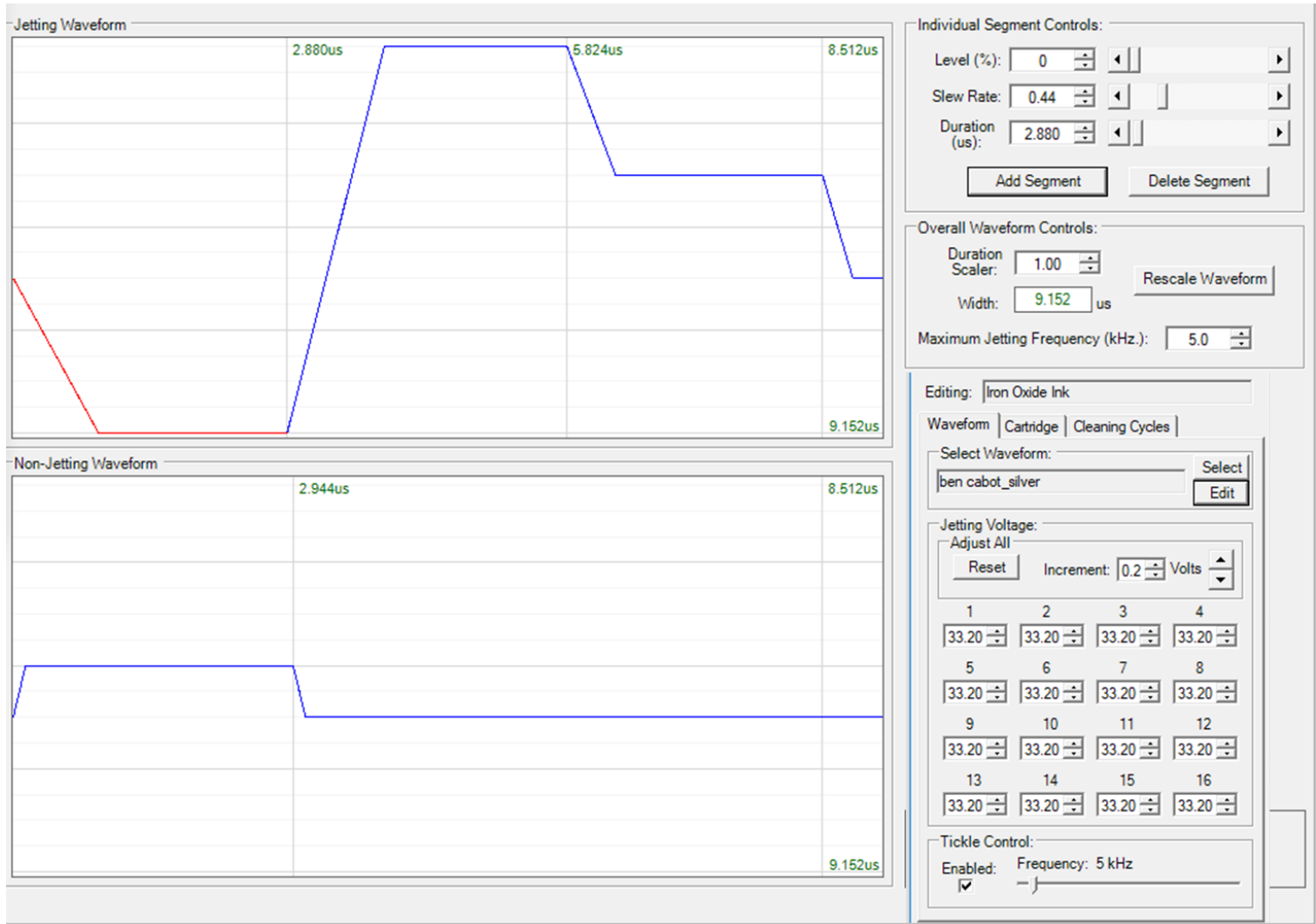

Figure S12. Jetting waveform for as-formulated iron oxide nanoparticles ink 